\title{
Single Nucleus Multiomic Profiling Reveals Age-Dynamic Regulation of Host Genes Associated with SARS-CoV-2 Infection
}

${ }^{1}$ Center for Epigenomics \& Department of Cellular \& Molecular Medicine, University of California, San Diego, La Jolla, CA 92093

2Biomedical Sciences Graduate Program, University of California San Diego, La Jolla, CA, 92093

${ }^{3}$ Department of Pediatrics, University of California-San Diego, La Jolla, CA 92093

${ }^{4}$ Division of Neonatology, Perinatal and Pulmonary Biology, Cincinnati Children's Hospital Medical Center, Cincinnati, OH 45267

${ }^{5}$ Department of Pediatrics, College of Medicine, University of Cincinnati, Cincinnati, $\mathrm{OH}$ 45267

${ }^{6}$ Vertex Pharmaceuticals, 3215 Merryfield Rd., San Diego CA, 92121

${ }^{7}$ Ludwig Institute for Cancer Research, La Jolla, CA 92093

${ }^{8}$ Laboratory of Genetics, Department of Medical Genetics, University of WisconsinMadison, Madison, WI 53706.

${ }^{9}$ Department of Pediatrics and Clinical \& Translational Science Institute, University of Rochester Medical Center

${ }^{10}$ Department of Biological Sciences, University of California-San Diego, La Jolla, CA 92093.

\#Authors contributed equally to this work. 
bioRxiv preprint doi: https://doi.org/10.1101/2020.04.12.037580; this version posted April 14,2020 . The copyright holder for this preprint (which

was not certified by peer review) is the author/funder, who has granted bioRxiv a license to display the preprint in perpetuity. It is made available under aCC-BY-NC-ND 4.0 International license.

47 Corresponding Authors*: Xin Sun (Lead contact)

48

xinsun@ucsd.edu

49

50

51

Sebastian Preissl

52

53

spreissl@health.ucsd.edu

54

Kyle J Gaulton

55

56

kgaulton@health.ucsd.edu

Allen Wang

57

a5wang@health.ucsd.edu 


\section{SUMMARY}

59 Respiratory failure is the leading cause of COVID-19 death and disproportionately 60 impacts adults more than children. Here, we present a large-scale snATAC-seq dataset 61 (90,980 nuclei) of the human lung, generated in parallel with snRNA-seq (46,500 nuclei), 62 from healthy donors of $\sim 30$ weeks, $\sim 3$ years and $\sim 30$ years of age. Focusing on genes 63 implicated in SARS-CoV-2 cell entry, we observed an increase in the proportion of 64 alveolar epithelial cells expressing ACE2 and TMPRSS2 in adult compared to young 65 lungs. Consistent with expression dynamics, 10 chromatin peaks linked to TMPRSS2 66 exhibited significantly increased activity with age and harbored IRF and STAT binding 67 sites. Furthermore, we identified 14 common sequence variants in age-increasing peaks 68 with predicted regulatory function, including several associated with respiratory traits and 69 TMPRSS2 expression. Our findings reveal a plausible contributor to why children are 70 more resistant to COVID-19 and provide an epigenomic basis for transferring this 71 resistance to older populations.

\section{Keywords:}

76 COVID-19, lung, SARS-CoV-2, single cell ATAC-seq, single cell RNA-seq, age dynamics, 77 ACE2, TMPRSS2, human sequence variants, interferon signaling pathway. 


\section{INTRODUCTION}

Aside from fulfilling gas-exchange functions that are vital for survival beginning with the first breath, the lung functions as a critical barrier to protect against inhaled pathogens such as viruses. As the COVID-19 pandemic swept across the world, the lung came into

82 focus because acute respiratory distress (ARDS) is the primary cause of mortality. Thus,

83 understanding how SARS-CoV-2 infects and impacts the lung has become an urgent call-

84 to-action.

85 The lung is composed of an elaborate airway tree that conducts air to and from the distal gas-exchange units called the alveoli. In an average human adult lung, an estimated 480 million alveoli give rise to approximately $1,000 \mathrm{ft}^{2}$ of gas-exchange surface area (Ochs et al., 2004). Airway and alveolar epithelium constitute the respiratory barrier that is exposed to inhaled pathogens. Respiratory epithelial cells are thereby at the frontline of infection, although pathogens that have bypassed the barrier can infect other cell types. The human airway epithelium is composed of luminal cells and basal cells. Luminal cells include club cells and goblet cells that moisturize the air and trap pathogens, as well as ciliated cells that sweep out inhaled particles. These luminal cells are underlined by basal cells, which serve as progenitors when luminal cells are lost after infection. The alveolar epithelium is composed of alveolar type 1 cells (AT1s) which line the gas-blood interface and alveolar type 2 cells (AT2s) which produce surfactant to reduce surface tension and protect against pathogens. While SARS-CoV-2 likely infects both the airway and alveolar regions of the lung, it is the damage to the alveolar region that underlines acute respiratory distress syndrome (Du et al., 2020).

Several large scale studies including efforts from LungMap and the Human Cell Atlas aim to generate a map of cell types in the human lung with single cell transcriptomics as the central modality (Reyfman et al., 2019; Schiller et al., 2019; Travaglini et al., 2020; Xu et al., 2016). Regions of the human genome, such as promoters or distal enhancers, can regulate cell-type specific gene expression in cis (Consortium, 2012; Roadmap Epigenomics et al., 2015; Thurman et al., 2012). Accessible or 'open' chromatin is a hallmark of cis-regulatory elements, and can be assayed using techniques such as

108 tissue heterogeneity single cell technologies like single cell ATAC-seq have been 
109 developed to map the epigenome and gene regulatory programs in component cell types 110 within heterogeneous tissues (Buenrostro et al., 2015; Chen et al., 2018; Cusanovich et 111 al., 2015; Cusanovich et al., 2018; Lareau et al., 2019; Satpathy et al., 2019). Profiles

112 derived from single cells can elucidate cell type-specific cis-regulatory elements, 113 transcriptional regulators driving element activity, and predicted target genes of distal 114 elements using single cell co-accessibility (Cusanovich et al., 2018; Lareau et al., 2019; 115 Pliner et al., 2018; Preissl et al., 2018; Satpathy et al., 2019). Human sequence variants 116 affecting susceptibility to complex physiological and disease traits are enriched in non117 coding sequence (Maurano et al., 2015; Pickrell, 2014), and cell type-specific profiles 118 derived from single cell epigenomic data can help prioritize cell types of action for these 119 variants (Chiou et al., 2019; Corces et al., 2020).

120 Both in silico structural modeling as well as biochemical assays have implicated 121 several key host proteins at the top of the hierarchy for SARS-CoV-2 infection. ACE2 has 122 been demonstrated as the receptor for not only the original SARS-CoV, but also SARS123 CoV-2 (Lan et al., 2020; Yan et al., 2020). Based mainly on literature from the original 124 SARS-CoV as well as emerging data from SARS-CoV-2 (Huang et al., 2006; Matsuyama 125 et al., 2020; Reinke et al., 2017; Walls et al., 2020; Zhou et al., 2016), TMPRSS2 and 126 CTSL are responsible for fusion of the virus with host cell by cleaving the viral Spike 127 protein. BSG is a receptor that can bind to the SARS-CoV spike protein (Chen et al., 128 2005) and SARS-CoV-2 contains a novel cleavage site for the protease Furin, adding 129 both genes to the list of host machinery highjacked by the virus (Coutard et al., 2020; 130 Walls et al., 2020). In this study, we will focus on the genes encoding these 5 proteins, 131 ACE2, TMPRSS2, CTSL, BSG, and FURIN, and determine their expression and 132 associated epigenomic landscape at single cell resolution in the non-diseased human 133 lung.

134 In the race to control the COVID-19 pandemic, there has been a tremendous collective 135 effort from the research community to elucidate the mechanism underlying SARS-CoV-2 136 infection. Our study contributes to this effort through a unique dataset profiling the human 137 lung. First, we generated single cell data across neonatal, pediatric, and adult lungs from 138 three donors in each group. These data allowed us to assess age-associated changes 139 with minimal technical variation. Second, from each lung sample, we generated parallel 
140 snRNA-seq and snATAC-seq data. This combination allowed us to associate cell type141 specific accessible chromatin profiles that may act as cis-regulatory regions that control 142 cell-type specific gene expression. Using these data, we first addressed cell-type 143 specificity and temporal dynamics of ACE2, TMPRSS2, CTSL, BSG, and FURIN 144 expression. We next identified candidate cis-regulatory elements co-accessible with the 145 promoters of these genes and characterized their cell-type specificity and temporal 146 dynamics. Finally, we profiled sequence variation that may impact cis-regulatory element 147 activity and contribute to differential susceptibility to SARS-CoV-2 infection.

148 Emerging epidemiology data, including on US cases reported by the CDC, 149 demonstrate that many fewer children tested positive for SARS-CoV-2 infection, and 150 those who tested positive generally show less severe symptoms than adults or elderly 151 individuals (Bi et al., 2020; CDC, 2020). This age divide coincides with the finding that 152 normal lung development in humans continues until the early 20s (Narayanan et al., 153 2012). Therefore COVID-19 preferentially impacts fully mature lungs relative to 154 developing lungs. Widespread speculation has attempted to explain these age155 associated differences, including immune senescence in the aging population. Defining 156 the mechanism underlying the apparent resistance of children to COVID-19 will inform 157 how we can transfer this resistance to adult and elderly populations.

\section{RESULTS}

\section{Single nucleus RNA-seq and ATAC-seq data generation}

163 To profile cell type specific gene expression and accessible chromatin dynamics in 164 the human lung, we performed single nucleus RNA-seq (snRNA-seq) and single nucleus 165 ATAC-seq (snATAC-seq) of non-diseased human lung tissue from donors of three age 166 groups: 30 week old gestational age (GA, prematurely born, 30wk ${ }^{\mathrm{GA}}$ ), $\sim 3$ year old (3yo), 167 and $\sim 30$ year old (30yo) (Supplementary Table 1). Three lungs were sampled for each 168 age group, with both males and females represented (Supplementary Table 1). Of the 9 169 donors, 5 were Caucasian, 1 was African American and 3 were of unknown ancestry. For 170 all samples, flash frozen biopsies from equivalent small airway regions of the lung were 
171 used. Nuclei were isolated from individual biopsies and split into two pools, one for 172 snRNA-seq and one for snATAC-seq. For snATAC-seq, we generated technical 173 replicates for one of the 3yo donors (D032) and an additional dataset for a lung sample 174 from a 4-month-old donor (Supplementary Table 1).

175 To generate snRNA-seq libraries, we used the droplet-based Chromium Single Cell 176 3' solution (10x Genomics) (Zheng et al., 2017). The datasets showed a clear separation 177 of nuclei from background in the knee plot (Figure S1A). The average number of nuclei 178 that passed initial quality control filtering per sample was 6,676 for $30 \mathrm{wk}^{\mathrm{GA}}, 7,379$ for 3 yo, 179 and 4,217 for 30yo (Figure S1B). Since we profiled nuclei with a high fraction of nascent, 180 unspliced RNA molecules, sequencing reads were mapped to an exon+intron reference. 181 We detected on average 1,662 gene/nuclei for $30 \mathrm{wk}^{\mathrm{GA}}, 1,394$ for 3yo and 1,260 for 30yo 182 (Figure S1C). Libraries were sequenced to comparable saturation (58.4\% for 30wk ${ }^{\mathrm{GA}}$, $18351.6 \%$ for $3 y 0$ and $55.0 \%$ for 30yo; Fig. S1D).

184 For snATAC-seq library generation we used a semi-automated combinatorial barcoding platform (Cusanovich et al., 2015; Fang et al., 2019; Preissl et al., 2018) . For each dataset, nuclei with >1,000 uniquely mapped sequencing reads were included in the 187 analysis (Fig. S1E). The average number of nuclei that passed this threshold per age 188 group was 8,691 for 30wk ${ }^{\mathrm{GA}}, 7,877$ for 3yo and 8,034 for 30yo (Fig. S1F). The average

189 number of reads per nucleus was 6,399 for $30 \mathrm{wk}^{\mathrm{GA}}, 7,199$ for 3 yo and 8,362 for 30 yo 190 (Fig. S1G). The fraction of reads in peaks (FRiP) on average per data set was $52.8 \%$ for $19130 \mathrm{wk}^{\mathrm{GA}}, 54.4 \%$ for 3yo and $45.6 \%$ for 30yo (Fig. S1H). These values indicate 192 consistently high signal to noise ratios for all libraries.

\section{Age-linked increase in host genes for SARS-CoV-2 entry}

195 In total, 46,500 single nucleus transcriptomes were included in the analysis after 196 filtering out low quality nuclei and potential barcode collisions (Figure S1, Supplementary 197 Table 2, see Methods). Following batch correction all datasets were merged, and 31 198 clusters were identified (Figure 1A). These clusters represented all major cell types in the 199 small airway region of the lung, as well as rare cell types such as pulmonary 200 neuroendocrine cells (Figure 1A, Figure S2A, Supplementary Table 2). We identified 20114,527 epithelial cells (31.2 \% of all nuclei) in our snRNA-seq dataset. This optimal 
202 representation and large number of cells allowed us power to profile gene expression 203 patterns of viral entry genes in lung epithelial cells. For downstream analysis, we excluded 204 an unclassified cluster, and enucleated erythrocytes because the latter were only 205 detected in a single neonatal sample, consistent with immaturity (Figure 1A).

206 Focusing on SARS-CoV-2 viral entry genes, we found that ACE2 transcript was 207 detected in very few nuclei (total 80 nuclei) in the normal lung and these nuclei were 208 enriched within the epithelial lineage (Figure 1B, Supplementary Table 2). Alveolar type 2092 (AT2) cells had the highest number of $A C E 2^{+}$nuclei, accounting for $48.8 \%$ of all ACE2210 expressing nuclei (39 out of total 80 ACE2 ${ }^{+}$nuclei). In comparison, TMPRSS2 transcript 211 was detected more frequently (e.g. in 3,315/7,226 nuclei, or $45.8 \%$ of the AT2 cells, 212 Figure 1C, Supplementary Table 2). Most TMPRSS2-expressing cells were epithelial 213 cells including alveolar type 1 and 2 (AT1, AT2) cells and airway cells such as club, 214 ciliated and goblet cells (Figure 1C, Supplementary Table 2). We also detected significant 215 correlation between the fraction of $\mathrm{ACE}^{+}$and TMPRSS2 ${ }^{+}$AT2 nuclei (Figure S2E) and 216 found 21 of the $39 \mathrm{ACE}^{+}$AT2 cells also expressed TMPRSS2 (Supplementary Table 2).

217 The other three candidate genes of SARS-CoV-2 host cell entry CTSL, BSG and FURIN 218 were expressed in a large number of AT1, AT2, matrix fibroblast, and M1 macrophage 219 cells, as well as a small number of additional cell types (Figure S2B-D, Supplementary 220 Table 2). These findings suggest that among cells that constitute the barrier exposed to 221 inhaled pathogens, cell types in both the airway and alveolar epithelium express genes 222 critical for SARS-CoV-2 entry.

223 We next asked if there were genes enriched in $A C E 2^{+}$AT2 cells as compared to ACE2224 AT2 cells to identify potentially co-expressed genes. Among genes that showed a trend 225 for higher expression in ACE2 $^{+}$compared to ACE2- cells was IFNGR1 (log2 (fold change) $226=0.4,-\log _{10}(p-$ value $)=5.0 ;$ FDR corrected $p=0.257$, Supplementary Table 3), raising the 227 possibility that ACE2 may be co-regulated with interferon pathway genes, in line with 228 conclusions of a recent study (Ziegler, 2020). In our data generated from normal lungs 229 this correlation was modest, suggesting there is low baseline co-expression of ACE2 and 230 IFNGR1. Among genes with increased expression in TMPRSS2 ${ }^{+}$versus TMPRSS2- AT2 231 cells was ICAM1 ( $\log 2$ (fold change)=0.27, $-\log 10($ FDR corrected $p)=12.2$, Supplementary 232 Table 3), which encodes a receptor for Rhinovirus (Zhou et al., 2017). The potential co- 
233 expression of TMPRSS2 and ICAM1 may contribute to the often-observed co-infection

234 by more than one respiratory virus. Indeed, co-infection of SARS-CoV-2 and other viruses

235 including Rhinovirus has been observed, promoting urgent calls to halt the clinical

236 practice of using positive test for other respiratory viruses as an indicator for the absence

237 of coronavirus infection (Wang et al., 2020; Wu et al., 2020). To gain additional insight

238 into the potential mechanisms of co-infection, we interrogated the expression of a number

239 of known factors, receptors and proteases that have been implicated in viral entry for 240 several key respiratory viruses (Figure S3)(Battles and McLellan, 2019; Bochkov and

241 Gern, 2016; Laporte and Naesens, 2017; Peck et al., 2015). For examples, consistent

242 with prior findings, we found that $C D H R 3$, a receptor for Rhinovirus C, was expressed

243 most abundantly in ciliated cells (Battles and McLellan, 2019; Bochkov and Gern, 2016;

244 Laporte and Naesens, 2017; Peck et al., 2015). ANPEP, the entry receptor for HCoV-

245 229E, was predominantly expressed in macrophages and to a lesser extent in club and

246 other epithelial cells (Waradon Sungnak, 2020; Yeager et al., 1992). Compared to ACE2,

247 DPP4, which encodes the host receptor for MERS-CoV, was detected much more

248 frequently overall, and especially in AT2, AT1 and T cells (Figure S3) (Raj et al., 2013;

249 Waradon Sungnak, 2020). This single cell resolved view may contribute to a

250 comprehensive map of the routes of respiratory viral entry.

251 The leading cause of death for COVID-19 is Acute Respiratory Distress Syndrome

252 (ARDS) which is characterized by failure of gas-exchange due to destruction of the 253 alveolar region of the lung (Du et al., 2020). AT2 is an abundant epithelial cell type in the 254 alveolar region and expresses all of the SARS-CoV-2 viral entry genes assayed here and 255 likely bears the brunt of infection. Consequently, we focused on AT2 cells for follow up 256 analysis. We found that the percentage of AT2 cells expressing ACE2 had an increasing 257 trend in 30yo adult samples compared to 3yo samples (Figure 1D). In addition, we found 258 a strong trend of increase in the percentage of AT2 cells expressing TMPRSS2 in adult 259 samples compared to 3yo samples $(41.2 \pm 6.6 \%$ for 3 yo and $57.4 \pm 7.7 \%$ for 30yo, p = 2600.05 (t-test), Figure 1E). While very few ACE2 $2^{+} T M P R S S 2^{+}$double positive AT2 nuclei 261 were detected, the fraction of these nuclei in all AT2s increased with age (0.2\% (6 nuclei) 262 in 30 $\mathrm{wk}^{\mathrm{GA}}, 0.3 \%$ (5 nuclei) in 3yo and 0.5\% (10 nuclei) in 30yo, Supplementary Table 2). 263 Of note, one of the samples in the $30 \mathrm{wk}^{\mathrm{GA}}$ cohort D062 appeared to be an outlier in its 
264 expression of multiple analyzed genes. A review of pathology notes revealed mild 265 features of respiratory distress syndrome including epithelial autolysis and increased 266 alveolar macrophages in this sample, suggesting potential reasons for the variation. In a 267 supplementary analysis, excluding this sample resulted in stronger age-associated 268 effects (Figure S2F, G). For example, there was a significant increase in the fraction of 269 TMPRSS2 $^{+}$AT2 cells between 30wk ${ }^{\mathrm{GA}}$ and 30yo samples (Figure S2G).

270 The increase in proportion of AT2 cells expressing ACE2 and TMPRSS2 is unlikely 271 due to differences in genes captured per nucleus as the adult samples had the lowest 272 numbers of genes/nucleus, suggesting that the extent of expression increase is likely a 273 conservative estimation (Figure S1C). In contrast to the percentage of AT2 nuclei 274 expressing these genes, the expression levels per nucleus were not different across 275 different age groups for either ACE2 (no nucleus had >1 UMI detected) or TMPRSS2 276 (Figure 1F). Together, an increased proportion of host cells expressed TMPRSS2 and 277 ACE2 in adults, the latter just a trend due to the sparsity of $A C E 2^{+}$cells, suggesting that 278 a higher percentage of cells in the adult lung can be infected by SARS-CoV-2.

279 Since a large proportion of COVID-19 patients are elderly, we sought to compare viral 280 entry gene expression in aged lungs to expression in our samples. The LungMap Human 281 Tissue Core, which provided the frozen biopsies for this study, does not have donors 282 older than $\sim 30$. We therefore instead, identified 4 publicly available scRNA-seq datasets 283 from non-diseased lungs of ages $>55$ that served as controls in pulmonary fibrosis studies 284 (Morse et al., 2019; Reyfman et al., 2019). We integrated snRNA-seq data from our study $285(n=9)$ with these 4 scRNA-seq samples (Supplementary Table 1) using Seurat 3 (Stuart 286 et al., 2019). AT2 cells clustered together across all samples with minimal evidence for 287 batch effects (Figure S4A). Compared to 30yo samples, we observed a trend for 288 increased frequency of $\operatorname{ACE}^{+}(p=0.095)$ and $\operatorname{TMPRSS}^{+}(p=0.070)$ AT2 cells in 289 the >55yo group (Aged; Figure S4B). While these patterns are consistent with 290 epidemiological findings that elderly are at highest risk, we make these observations 291 cautiously due to the multiple potential confounding variables present when comparing 292 across independent datasets spanning multiple methodologies. 
Annotation of cis-regulatory sequences linked to SARS-CoV-2 viral entry gene activity

To investigate cis-regulatory elements driving cell-type specific and age-related patterns of SARS-CoV-2 viral entry gene expression, we examined SnATAC-seq data 298 generated from the same nuclei preparations. After batch correction and filtering of low299 quality nuclei and likely doublets, we clustered and analyzed a total of 90,980 single 300 nucleus accessible chromatin profiles. We identified 19 clusters representing epithelial 301 (AT2, AT2, club, ciliated, basal and neuroendocrine), mesenchymal (myofibroblast, 302 pericyte, matrix fibroblast 1 and matrix fibroblast 2), endothelial (arterial, lymphatic, and 3032 clusters of capillaries), and hematopoietic cell types (macrophage, B-cell, T-cell, NK cell 304 and enucleated erythrocyte) (Figure 2A). Supporting these cluster annotations, we observed cell type-specific patterns of chromatin accessibility at known marker genes for each cell type (Figure S5A).

Focusing on SARS-Cov-2 viral entry genes, both ACE2 and TMPRSS2 were primarily accessible throughout their gene body in alveolar cells such as AT1, AT2, and airway cells such as club, ciliated, and basal cells (Figure 2B). Conversely, the CTSL gene body exhibited chromatin accessibility across epithelial cells, mesenchymal cells, endothelial, and macrophages. BSG and FURIN also showed broad chromatin accessibility patterns 312 with the highest activity in endothelial cells, such as capillaries (Figure 2B). Overall, the 313 patterns of chromatin accessibility across cell types at genes involved in SARS-CoV-2 314 cell entry substantiate our conclusions from snRNA-seq data, including the finding that ACE2 and TMPRSS2 are primarily expressed in alveolar and airway cells (Figure 1B,C).

To identify specific cis-regulatory elements that might control cell type-restricted 318 each cell type and called accessible chromatin sites from the aggregated profiles using 319 MACS2 (Zhang et al., 2008). We then identified sites mapping within $650 \mathrm{~kb}$ of each 320 SARS-CoV-2 viral entry gene, and further identified sites that were co-accessible with the 321 gene promoter using Cicero (Pliner et al., 2018). At the ACE2 locus, we identified 165 322 accessible chromatin sites mapping within the $\pm 650 \mathrm{~kb}$ window (Figure $2 \mathrm{C}$, 323 Supplementary Table 4). Of these 165 sites, only two were co-accessible with the ACE2 324 promoter (Figure 2C, Supplementary Table 5). We speculate that the low number of co- 
325 accessible sites is likely due to the small percentage of $A C E 2^{+}$nuclei (Figure $\left.1 \mathrm{~B}\right)$. When

326 examining the accessibility of the 165 peaks at the ACE2 locus across cell types, we

327 observed clear sub-groupings of sites, including those specific to basal cells, specific to

328 ciliated cells, and shared across basal, ciliated, AT1, AT2, and club cells (Figure 2C,

329 Supplementary Table 5).

330 At the TMPRSS2 locus, we identified 289 accessible chromatin sites mapping in the $331 \pm 650 \mathrm{~kb}$ window, of which 37 were co-accessible with the TMPRSS2 promoter (Figure 2D,

332 Supplementary Tables 4 and 5). In agreement with TMPRSS2 gene accessibility in 333 alveolar and airway cells, 113 out of the 289 elements exhibited patterns of accessibility 334 specific to basal, ciliated, club, AT1, and AT2 cells. We observed a basal cell-specific 335 cluster and two broader epithelial cell clusters (basal, ciliated, and club enriched; and 336 club, AT1, and AT2 enriched) (Figure 2D, Supplementary Table 5). Notably, the majority 337 of sites co-accessible with TMPRSS2 (25/37) were found within these broad alveolar- and 338 airway-enriched clusters suggesting that these elements are likely responsible for 339 alveolar and airway expression of TMPRSS2.

340 Finally, at the CTSL, FURIN, and BSG loci we identified 262, 293, and 272 accessible 341 chromatin sites, respectively, within a $\pm 650 \mathrm{~kb}$ window of which 6,56 , and 47 were co342 accessible with their respective gene promoters (Figure S5B, C, D, Supplementary 343 Tables 4 and 5). Sites for all three genes exhibited broad patterns of accessible chromatin 344 signal across cell types consistent with broad accessibility across gene bodies. This 345 collection of cell-type resolved candidate cis-regulatory elements associated with SARS346 CoV-2 host genes will be critically important for follow up studies to determine how host 347 cell genes are regulated and how genetic variation within these elements contributes to 348 infection rate and disease outcomes.

Cis-regulatory elements linked to TPMRSS2 are part of an age-related regulatory program associated with immune signaling in AT2 cells

Having observed increasing percentages of TMPRSS2 expressing cells with age in 353 AT2 cells (Figure 1E, Figure S2G), we speculated that TMPRSS2 may be under the 354 control of an age-related cis regulatory program. To investigate whether an ageassociated cis-regulatory network exists in AT2 cells, we identified accessible chromatin 
356 sites in AT2 cells that show dynamic accessibility across donor age groups. Based on our

357 findings from snRNA-seq we speculate that these dynamics will be at least in part due to

358 a higher number of cells expressing these genes rather than more activity within a cell.

359 We tested all possible pairwise age comparisons between AT2 signal from each of the 360 three groups of $30 \mathrm{wk}^{\mathrm{GA}}$, 3yo, and 30yo donors while accounting for donor to donor 361 variability (Figure 3A). Overall, we identified 22,745 age-linked sites in AT2 cells which 362 exhibited significant differences (FDR<0.05) in any pairwise comparison (Figure $3 A, B)$. 363 Clustering of these dynamic peaks revealed five predominant groups of age-dependent 364 chromatin accessibility patterns (cl-cV, Fig 3B).

365 We identified two clusters of AT2 sites exhibiting increasing accessibility with age 366 including several sites at candidate genes for SARS-CoV-2 host genes (cIll 30yo enriched 367 and clV 3yo + 30yo) (Figure 3B, Figure S6A, B). Intriguingly, these two clusters were 368 enriched for processes related to viral infection and immune response such as viral 369 release from host cell, interferon-gamma mediated signaling pathway, and positive 370 regulation of ERBB signaling pathway (Figure 3C, Supplementary Table 6). Also, these 371 age-dependent clusters were also enriched for phenotypes substantiated in mouse 372 studies, such as pulmonary epithelial necrosis, increased monocyte cell number, and 373 chronic inflammation (Fig. 3C, Supplementary Table 6). Further supporting an immune 374 association with age-related chromatin accessibility in AT2 cells, we observed an 375 enrichment of sequence motifs within these clusters for transcription factors involved in 376 immune signaling such as STAT, IRF, and FOS/JUN (Figure 3D, Supplementary Table 377 7).

378 We focused on the TMPRSS2 locus and determined how many of the 37 accessible 379 chromatin sites co-accessible with the TMPRSS2 promoter (in Figure 2D) showed 380 increased accessibility with age in AT2 cells. We identified 13 sites with age-increased 381 accessibility, of which 10 had significant effects (FDR $<0.05$ via EdgeR and/or $p<0.05$ 382 via t-test) (Figure 3E, F, Figure S6, Supplementary Table 5). Age-increasing sites linked 383 to TMPRSS2 harbored sequence motifs for transcription factors such as NKX, FOXA, 384 CEBPA, and inflammation-related factors such as STAT, IRF, and FOS/JUN (Figure 3G) 385 many of which were corroborated by available ChIP-seq data in lung related samples (Oki 386 et al., 2018). Furthermore, at 12 of the 13 age-increasing sites, we uncovered additional 
387 evidence for enhancer-related histone modifications from ENCODE supporting that they

388 have cis-regulatory activity (Figure 3H) (Consortium, 2012). When viewed in genomic

389 context these sites showed a clear age-dependent increase in read depth likely reflecting

390 a higher fraction of accessible nuclei (Figure 3I).

Genetic variants predicted to affect age-increased TMPRSS2 sites are associated with respiratory phenotypes and TMPRSS2 expression

Mapping the discrete accessible chromatin sites at genes required for SARS-CoV-2 viral entry allowed us to next characterize non-coding sequence variation that might affect regulation of these sites and contribute to phenotypic differences in the risk of lung disease. In particular, we focused on the 37 sites linked to TMPRSS2 activity including 13 with age-increased chromatin accessibility.

399 In total, 8,002 non-singleton sequence variants in the gnomAD v3 database 400 (Karczewski et al., 2019) overlapped a site either linked to or within 250kb of the 401 TMPRSS2 promoter. To determine which of these variants might affect regulatory activity 402 in AT2 cells, we applied a machine learning approach (deltaSVM) (Lee et al., 2015) to 403 model AT2 chromatin accessibility and predict variants with allelic effects on chromatin 404 (see Methods). We identified 721 variants with significant effects (FDR<0.1) on AT2 405 chromatin accessibility, of which 148 mapped in an age-dependent site linked to 406 TMPRSS2 (Figure 4A). Among these 148 variants, 14 were common (defined here as 407 minor allele frequency $>1 \%$ ) in at least one major population group in gnomAD, several 408 of which were predicted to disrupt AT2 age-dynamic TF motifs such as FOS/JUN, IRF, 409 STAT, RUNX, NKX and ESR1 (Figure 4A). The common variants generally had 410 consistent frequencies across populations, except for rs35074065 which was much less 411 common in East Asians (EAS) relative to other populations (MAF=0.005, Figure 4B).

412 We next determined whether common variants with predicted AT2 regulatory effects 413 were associated with phenotypes related to respiratory function, infection, medication use 414 or other traits using GWAS data generated using the UK Biobank (UKBB) (Sudlow et al., 415 2015). Across the 11 variants tested for association in UKBB data, the most significant 416 association was between rs35074065 and emphysema ( $\left.p=5.64 \times 10^{-7}\right)$ (Figure 4C). This 417 variant was also more nominally associated $(p<0.005)$ with asthma $\left(p=6.7 \times 10^{-4}\right)$ and 
418 influenza vaccine $\left(p=1.76 \times 10^{-3}\right)$. Furthermore, the majority of tested variants $(8 / 11)$ were

419 nominally associated $\left(\mathrm{p}<1 \times 0^{-3}\right)$ with at least one phenotype related to respiratory function

420 or respiratory medication use including salmeterol + fluticasone propionate, which is

421 commonly used to treat asthma and COPD ( $r$ 7279188 p=1.3 $\left.\times 10^{-5}\right)$, bacterial pneumonia $422\left(\right.$ rs2838089 $\left.\mathrm{p}=2.4 \times 10^{-4}\right)$, bronchiectasis $\left(\mathrm{rs} 9974995 \mathrm{p}=7.1 \times 10^{-4}, r \mathrm{~s} 568517 \mathrm{p}=8.1 \times 10^{-4}\right)$,

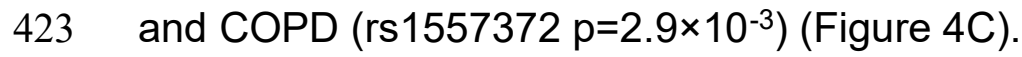

424 Given that common AT2 variants showed predicted regulatory function and 425 association with respiratory disease and infection phenotypes, we next asked whether 426 these variants regulated the expression of TMPRSS2 using human lung eQTL data from 427 the GTEx v8 release. Among variants tested for association in GTEx, we observed a 428 highly significant eQTL for TMPRSS2 expression at rs35074065 $\left(p=3.9 \times 10^{-11}\right)$ as well as 429 more nominal eQTL evidence at $r$ 1557372 $\left(p=2.9 \times 10^{-5}\right)$ and $r$ 9974995 $\left(p=3.5 \times 10^{-6}\right)$. 430 Furthermore, in fine-mapping data, rs35074065 had a high posterior probability $431 \quad(P P A=41.6 \%)$ and therefore likely has a direct casual effect on TMPRSS2 expression 432 (Figure 4D). This variant further disrupted sequence motifs for IRF and STAT transcription 433 factors, suggesting that its effects may be mediated through interferon signaling and anti434 viral programs (Figure 4D).

435 As the TMPRSS2 eQTL at rs35074065 was identified in bulk lung samples, we finally 436 sought to determine the specific cell types driving the effects of this eQTL. Using cell type437 specific expression profiles derived from our snRNA-seq data, we estimated the 438 proportions of 14 different cell types present in the 515 bulk lung RNA-seq samples from 439 GTEx v8 (Figure 4E) (Aguet et al., 2019). We then tested the association between 440 rs35074065 and TMPRSS2 expression while including estimated cell type proportions for 441 each sample in the eQTL model (see Methods). We observed highly significant 442 association when including AT2 cell proportion $\left(p=3.8 \times 10^{-18}\right)$ as well as macrophage 443 proportion $\left(p=4.0 \times 10^{-12}\right)$, supporting the possibility that the TMPRSS2 eQTL at 444 rs35074065 acts through AT2 cells and macrophages (Figure 4F). 
In this study, we focused on the lung, the organ at the center of COVID-19 morbidity and mortality. We generated a snATAC-seq reference dataset of the healthy human lung

450 at three postnatal stages, and in parallel generated snRNA-seq data from the same 451 samples to allow comparison with gene expression. Importantly, datasets were produced 452 using uniform tissue procurement and single nucleus technologies for both modalities 453 across samples. This consistency allowed us to uncover age-associated dynamics in 454 gene expression and regulation. While we focus on COVID-19 related genes in this study, 455 the datasets more broadly enable in-depth analysis of cell-type resolved dynamics of 456 chromatin accessibility and gene expression in the human lung. We hope these datasets 457 will be further utilized by the community to enhance knowledge and treatment of lung 458 diseases.

459 One of the strongest findings that has been corroborated by multiple large-scale 460 epidemiological studies is that infants and children, while still susceptible to infection, 461 generally do not develop symptoms as severe as adults (Bi et al., 2020; CDC, 2020). 462 Although the underlying molecular basis of this skew is unclear and is likely multifactorial, 463 our data demonstrate that $A C E 2^{+}$and TMPRSS2 ${ }^{+}$and $A C E 2^{+} / T M P R S S 2^{+}$are detected 464 in a higher proportion of AT2 nuclei in adult samples compared to the younger samples. 465 These findings suggest that SARS-CoV-2 may enter proportionally fewer cells in younger 466 lungs compared to adult lungs, leading to tempered viral replication and damage. While 467 we await clinical validation of this finding, this difference in viral entry factors, in addition 468 to likely differences in immune response to viral infection, may explain the age-related 469 bias in COVID-19 severity.

470 The observed increase in the proportion of cells expressing viral entry genes is further 471 corroborated by age-related changes in accessible chromatin, which offers insight for 472 using gene regulatory mechanisms to restrict the expression of viral entry genes. For 473 example, at the TMPRSS2 locus we identified 10 accessible chromatin sites that showed 474 significantly increased accessibility with age. These sites may therefore represent cis 475 regulatory elements that contribute to activation of TMPRSS2 gene expression in an 476 increasing number of cells in adults and represent possible sites to modulate in order to 477 restrict expression. Furthermore, one of the age-dependent sites harbors a sequence 
variant (rs35074065) significantly associated with TMPRSS2 expression and respiratory phenotypes, suggesting it may be of particular value in this context.

To explore potential avenues for manipulating the expression of viral entry genes, we identified transcription factors enriched in sites with increased chromatin accessibility in adult AT2 cells compared to younger AT2 cells. These included transcription factors involved in stress and immune responses. For example, key interferon pathway-related factors STAT and IRF have binding sites in the 10 age-increased TMPRSS2 peaks. The likely causal TMPRSS2 eQTL variant rs35074065 is predicted to disrupt STAT and IRF binding, raising the possibility that STAT and/or IRF binding at this site may directly control TMPRSS2 gene expression.

While our findings suggest that interferon pathway transcription factors may play a role in regulating the expression of SARS-CoV-2 entry genes such as TMPRSS2, extensive preclinical studies are needed to validate this regulation in an in vivo context. As a key anti-viral factor, interferon is stimulated in host cells upon infection by viruses, likely including SARS-CoV-2 (Lukhele et al., 2019; Mesev et al., 2019; Xia et al., 2018). The literature contains conflicting data regarding whether and how viral infection may act through the interferon pathway to regulate viral entry gene expression. For example, binding of the original SARS-CoV spike protein to ACE2 receptor in mice led to reduced Ace2 expression in the lung (Kuba et al., 2005). However, a recent single-cell study suggested that viral-induced interferon activation stimulates ACE2 expression (Ziegler, 2020). We caution that the potential effect of interferon signaling on COVID-19 needs to be investigated beyond viral entry, as the pathway likely has distinct roles in the different phases of the disease.

In our lung snRNA-seq data, ACE2 is detected in a very small number of cells, a finding that is corroborated by a number of recent single cell studies (Qi et al., 2020;

504 fraction of nuclei that are ACE2 positive could be due to low overall expression which in 505 turn results in significant dropout in single cell or single nucleus RNA-seq. This suggests 506 the possibility that ACE2 may not be needed at high levels for viral attachment to host 507 cells. Alternatively, it is plausible that alternative receptors such as BSG also facilitate 508 SARS-CoV-2 attachment in vivo. Compared to ACE2, BSG is expressed and co- 
509 expressed with proteases in a higher fraction of nuclei in AT2 and in additional cell types 510 in the human lung.

511 To limit SARS-CoV-2 infection by manipulating the expression of viral entry proteins, 512 we caution that inhibiting ACE2 expression should not be a recommended strategy. Aside

513 from being a viral receptor gene, ACE2 is also required for protecting the lung from injury514 induced acute respiratory distress phenotypes, the precise cause of COVID-19 mortality 515 (Imai et al., 2005). Thus, inhibiting ACE2 expression may compromise the ability of the 516 lung to sustain damage. In comparison, Tmprss2 mutant mice show no defects at 517 baseline and are more resistant to the original SARS-CoV infection (Iwata-Yoshikawa et 518 al., 2019; Kim et al., 2006). Thus, manipulating the expression of genes such as 519 TMPRSS2 may represent a safer path to limit SARS-CoV-2 viral entry. TMPRSS2 is also 520 involved in the entry of other respiratory viruses such as influenza, suggesting that 521 modulating its expression may also be effective in deterring entry and spread of other 522 viruses (Limburg et al., 2019).

523 In this study, we present the first snATAC-seq dataset of the human lung and 524 complementary snRNA-seq data from the same samples. Here, we used COVID-19 525 genes to demonstrate how this dataset can be utilized. As COVID-19 GWAS data 526 emerge, our datasets will offer a powerful cell type-resolved platform to interrogate 527 mechanisms that may underlie genetic differences in the susceptibility and response to 528 SARS-CoV-2 infection. Furthermore, our results suggest that modulation of the interferon 529 pathway is a possible avenue to restrict TMPRSS2 expression and viral entry. 530 Identification of regulators that restrict the expression of viral entry genes without 531 detrimentally affecting other aspects of the normal antiviral response will be a safe and 532 effective strategy towards combating COVID-19. We note that this work is a product of 533 the NHLBI-funded LungMap consortium, and our joint goal is to provide the community 534 with fundamental knowledge of the human lung to help combat COVID-19. 


\section{ACKNOWLEDGEMENTS}

We are extremely grateful to the families who have generously given such precious gifts to support this research. We thank all the members of the LungMAP Consortium for their collaborations. We thank Dr. Bing Ren, Dr. Maike Sander, members of the Sun lab,

541 Gaulton lab, Ren lab and the UCSD Center for Epigenomics for insightful discussions.

542 We thank S. Kuan for sequencing and B. Li for bioinformatics support. We thank K. 543 Jepsen and the UCSD IGM Genomics Center for sequencing the snRNAseq libraries. We 544 thank the QB3 Macrolab at UC Berkeley for purification of the Tn5 transposase.

Funding: This work was supported by NHLBI Molecular Atlas of Lung Development

546 Program Research Center grant 1 U01 HL148867 (to X.Sun, S. Preissl, A. Wang and J.

547 Verheyden), and Human Tissue Core grant U01HL122700 / HL148861 (to G.H. Deutsch,

548 T.J. Mariani, G.S. Pryhuber). Work at the Center for Epigenomics was supported in part 549 by the UC San Diego School of Medicine.

\section{AUTHOR CONTRIBUTIONS}

Conceptualization: A.W., K.J.G., S.P., X.S.; Methodology: A.W., K.J.G., S.P., K.Z.; 554 Validation: J.M.V., R.E.Y., J.B.; Formal analysis: A.W., K.J.G., S.P., J.C., O.B.P., J.B., 555 M.J.V., J.M.N., M.G., Y.X.; Investigation: J.B., J.M.V., X.H.; Resources: R.M., H.H., L.R., 556 C.P., G.P., J.A.W.; Data curation: A.W., K.J.G., S.P., J.C., O.B.P., J.B., M.J.V., P.K., 557 D.A.F; Writing: A.W., J.C., J.M.V., K.J.G., S.P., X.S.; Supervision: A.W., K.J.G., S.P., 558 J.A.W., X.S.; Project administration: A.W., K.J.G., S.P., J.M.V., X.S.; Funding acquisition: 559 A.W., K.J.G., S.P., J.M.V., X.S. 
Figure 1. snRNA-seq of human lungs reveals expression of SARS-CoV-2 cell entry genes in the epithelial cell lineage. A UMAP embedding and clustering result of 46,500 snRNA-seq data from 9 donors (Premature born (30 week ${ }^{\mathrm{GA}}$ of pregnancy), 3 yo, 30 yo; $\mathrm{n}=3$ per time point) identifies 31 clusters. Each dot represents a nucleus. Spread-out grey dots correspond to nuclei of unclassified cluster. B, C Cluster specific violin blots of gene expression of B ACE2 and C TMPRSS2. D, E Fraction of AT2 cells with expression of ACE2 and TMPRSS2 at each time point. All data are represented as mean \pm SD. $p$ values derived from t-tests; One-way ANOVA did not reach significance. F Box plot of log normalized expression of TMPRSS2 in AT2 cells at each time point. Displayed are the median expression values for AT2 nuclei in individual samples with at least $1 \mathrm{UMI}$.

Figure 2. snATAC-seq analysis of human lungs reveals candidate cis regulatory elements for ACE2 and TMPRSS2. A UMAP embedding and clustering results of snATAC-seq data from 90,980 single-nucleus chromatin profiles from ten donors (Premature born (30 week $\left.{ }^{\mathrm{GA}}, \mathrm{n}=3\right), 4$ month old $(\mathrm{n}=1), 3$ yo $(\mathrm{n}=3)$ and 30 yo $(\mathrm{n}=3)$ ). B Gene accessibility of candidate SARS-CoV-2 cell entry genes. C Union set of peaks identified in all clusters surrounding ACE2 (+/- $650 \mathrm{~kb})$ and elements that show coaccessibility (co-accessibility score $>0.05$ ) with the ACE2 promoter via Cicero (Cusanovich et al., 2018) (top panel). Hierarchical clustering of the relative proportion of cells (see methods) with a fragment within 165 peak regions surrounding ACE2 (lower panel). Asterisks highlight peaks co-accessible with the ACE2 promoter via Cicero. Horizontal red box highlights peaks with increased relative accessibility shared in basal, ciliated, AT1, AT2 and club cells as compared to other cell types. Vertical red box highlights peaks with increased relative accessibility in AT2 cells. D Union set of peaks identified in all clusters surrounding TMPRSS2 $(+/-650 \mathrm{~kb})$ and elements that show coaccessibility with the TMPRSS2 promoter (top panel; co-accessibility score $>0.05$

589 (Cusanovich et al., 2018)). Hierarchical clustering of the relative proportion of cells with a 590 fragment within 289 peak regions surrounding TMPRSS2 (lower panel). Horizontal red 591 box highlights peaks with increased relative accessible cells shared in basal, ciliated, 
592 club, AT1 and AT2 cells as compared to other cell types. Vertical red box highlights peaks

593 with increased relative accessibility in AT2 cells. Asterisks highlight peaks co-accessible

594 with the TMPRSS2 promoter.

Figure 3. Age-increasing accessible chromatin in AT2 cells exhibits signatures of immune regulation and harbors TMPRSS2-linked sites of chromatin accessibility.

A Differential analysis was performed on AT2 cells using pairwise comparisons between three ages with replicates ( $n=3$ per stage). B K-means cluster analysis $(K=5)$ of relative accessibility scores (see Methods) for 22,845 age-dynamic peaks (FDR < 0.05, EdgeR) in AT2 cells. Clusters III and IV show increasing accessibility with age and contain seven TMPRSS2-co-accessible sites. C GREAT (McLean et al., 2010) analysis of elements in group clll (left panel) and cIV (right panel) shows enrichment of immune related gene ontology terms. D Transcription factor motif enrichment analysis of elements in clll and cIV. E Classification of age-dynamic patterns across the 37 TMPRSS2-co-acessible sites based on the relative percentage of AT2 cells with at least one fragment overlapping each peak. Red bars indicate dynamic peaks identified from analysis in B (FDR < 0.05, EdgeR). accessibility in AT2 with aging (top panel in 3E). Black asterisk, $p<0.05$ (T-test); Red asterisk, FDR < 0.05 (EdgeR) from dynamic peak analysis in B. G Annotation of motifs

611 and evidence for transcription factor association within age-increased peaks. Blue bar, 612 Motif present (FIMO); Green bar, Motif present (FIMO) and transcription factor 613 association (ChIP-Atlas). H Overlap with ENCODE histone modification ChIP-seq data

614 (Consortium, 2012) from SCREEN. I Genome browser representation of four TMPRSS2615 linked peaks across age groups.

617 Figure 4. Genetic variants predicted to affect age-increasing AT2 accessible 618 chromatin are associated with respiratory phenotypes and TMPRSS2 expression.

619 A Top: genome browser view of sites linked to TMPRSS2 activity including those with 620 age-dependent increase in activity. Right: Non-singleton genetic variants in gnomAD v3 621 mapping in each age-dependent site with predicted effects (FDR<.10) on AT2 chromatin 622 accessibility using deltaSVM. Variants within each site are organized based on whether 
623 the reference (ref) or alternate (alt) allele has a higher predicted effect. Left: DeltaSVM

624 scores of variants with predicted effects on AT2 chromatin accessibility and common

625 (defined as MAF>1\%) in at least one major population group in gnomAD v3, annotated

626 with sequence motifs overlapping the variant for TF families enriched in age-increased

627 AT2 sites. B Population frequency of variant rs35074065, which had predicted AT2

628 effects and was present at much lower frequency in East Asians relative to other

629 population groups. AFR: African, AMR: Latino/American, ASJ: Ashkenazi Jewish, EAS:

630 East Asian, FIN: Finnish, EUR: European (non-Finnish). C Association of common

631 variants with predicted AT2 effects with human phenotypes in the UK Biobank. The

632 majority of tested variants show at least nominal evidence $(p<0.005)$ for association with

633 phenotypes related to respiratory disease, infection and medication. D Fine-mapping

634 probabilities for an TMPRSS2 expression QTL in human lung samples from the GTEx

635 project release v8. The variant rs35074065 has the highest casual probability (PPA=.42)

636 for the eQTL, maps in an age-dynamic AT2 site and is predicted to disrupt binding of IRF

637 and STAT TFs. Variants are colored based on r2 with rs35074065 in 1000 Genomes

638 Project data using all populations. E Estimated cell type proportions for 515 human lung

639 samples from GTEx derived using cell type-specific expression profiles for cell types with

640 more than 500 cells from snRNA-seq data generated in this study. F Association $p$-values

641 between rs35074065 genotype and TMPRSS2 lung expression after including an

642 interaction term between genotype and estimated cell type proportions for each sample.

643 We observed stronger eQTL association when including an interaction with AT2 cell

644 proportion as well as macrophage proportion. 
METHODS

\section{Human subjects and tissue collection}

648 Donor lung samples were provided through the federal United Network of Organ Sharing via National Disease Research Interchange (NDRI) and International Institute for Advancement of Medicine (IIAM) and entered into the NHLBI LungMAP Biorepository for Investigations of Diseases of the Lung (BRINDL) at the University of Rochester Medical Center overseen by the IRB as RSRB00047606, as previously described (Ardini-Poleske et al., 2017; Bandyopadhyay et al., 2018). Portions $\left(0.25-1.0 \mathrm{~cm}^{3}\right)$ of small airway region of right middle lobe (RML) lung tissue were frozen in cryovials over liquid nitrogen and placed at $-80^{\circ} \mathrm{C}$ for storage. Upon request, while kept frozen on dry ice, a tissue piece

656 (approximately $100 \mathrm{mg}$ ) was chipped off the sample. These smaller samples were then shipped in cryovials to UCSD on an abundance of dry ice.

\section{Single nucleus ATAC-seq data generation}

Combinatorial barcoding single nucleus ATAC-seq was performed as described previously with modifications (Cusanovich et al., 2015; Fang et al., 2019; Preissl et al., 662 2018) and using new sets of oligos for tagmentation and PCR (Supplementary Table 8).

663 Briefly, for each sample, lung tissue was homogenized using mortar and pestle on liquid 664 nitrogen. $1 \mathrm{ml}$ nuclei permeabilization buffer (10mM Tris-HCL (pH 7.5), 10mM NaCl, 3mM $665 \mathrm{MgCl}$, $0.1 \%$ Tween-20 (Sigma), 0.1\% IGEPAL-CA630 (Sigma) and 0.01\% Digitonin 666 (Promega) in water (Corces et al., 2017)) was added to $30 \mathrm{mg}$ of ground lung tissue and 667 tissue was resuspended by pipetting for 8-15 times. Nuclei suspension was incubated for $66810 \mathrm{~min}$ at $4^{\circ} \mathrm{C}$ and filtered with $30 \mu \mathrm{m}$ filter (CellTrics). Nuclei were pelleted with a swinging 669 bucket centrifuge (500 x g, $5 \mathrm{~min}, 4^{\circ} \mathrm{C} ; 5920 \mathrm{R}$, Eppendorf) and resuspended in $500 \mu \mathrm{L}$ 670 high salt tagmentation buffer $(36.3 \mathrm{mM}$ Tris-acetate $(\mathrm{pH}=7.8), 72.6 \mathrm{mM}$ potassium671 acetate, $11 \mathrm{mM} \mathrm{Mg-acetate,} \mathrm{17.6 \%} \mathrm{DMF)} \mathrm{and} \mathrm{counted} \mathrm{using} \mathrm{a} \mathrm{hemocytometer.}$ 672 Concentration was adjusted to 2,000 nuclei/9 $\mu$ l, and 2,000 nuclei were dispensed into 673 each well of one 96-well plate. For tagmentation, $1 \mu \mathrm{L}$ barcoded Tn5 transposomes (Fang 674 et al., 2019) was added using a BenchSmart ${ }^{\text {TM }} 96$ (Mettler Toledo), mixed five times and 675 incubated for $60 \mathrm{~min}$ at $37^{\circ} \mathrm{C}$ with shaking (500 rpm). To inhibit the Tn5 reaction, $10 \mu \mathrm{L}$ 
676 of 40 mM EDTA were added to each well with a BenchSmart ${ }^{\text {TM }} 96$ (Mettler Toledo) and 677 the plate was incubated at $37{ }^{\circ} \mathrm{C}$ for $15 \mathrm{~min}$ with shaking (500 rpm). Next, $20 \mu \mathrm{L} 2 \times$ sort 678 buffer (2 \% BSA, 2 mM EDTA in PBS) was added using a BenchSmart ${ }^{\text {TM }} 96$ (Mettler 679 Toledo). All wells were combined into a FACS tube and stained with $3 \mu \mathrm{M}$ Draq7 (Cell 680 Signaling). Using a SH800 (Sony), 20 2n nuclei were sorted per well into eight 96-well 681 plates (total of 768 wells) containing $10.5 \mu \mathrm{L}$ EB (25 pmol primer i7, 25 pmol primer i5, $682200 \mathrm{ng}$ BSA (Sigma). Preparation of sort plates and all downstream pipetting steps were 683 performed on a Biomek i7 Automated Workstation (Beckman Coulter). After addition of 1 $684 \mu \mathrm{L} 0.2 \%$ SDS, samples were incubated at $55^{\circ} \mathrm{C}$ for $7 \mathrm{~min}$ with shaking (500 rpm). $1 \mu \mathrm{L}$ $68512.5 \%$ Triton-X was added to each well to quench the SDS. Next, $12.5 \mu \mathrm{L}$ NEBNext High686 Fidelity $2 \times$ PCR Master Mix (NEB) were added and samples were PCR-amplified $\left(72{ }^{\circ} \mathrm{C}\right.$ $6875 \mathrm{~min}, 98^{\circ} \mathrm{C} 30 \mathrm{~s},\left(98^{\circ} \mathrm{C} 10 \mathrm{~s}, 63^{\circ} \mathrm{C} 30 \mathrm{~s}, 72^{\circ} \mathrm{C} 60 \mathrm{~s}\right) \times 12$ cycles, held at $\left.12{ }^{\circ} \mathrm{C}\right)$. After 688 PCR, all wells were combined. Libraries were purified according to the MinElute PCR 689 Purification Kit manual (Qiagen) using a vacuum manifold (QIAvac 24 plus, Qiagen) and 690 size selection was performed with SPRI Beads (Beckmann Coulter, 0.55x and 1.5x). 691 Libraries were purified one more time with SPRI Beads (Beckmann Coulter, 1.5x). 692 Libraries were quantified using a Qubit fluorimeter (Life technologies) and the 693 nucleosomal pattern was verified using a Tapestation (High Sensitivity D1000, Agilent).

694 The library was sequenced on a HiSeq4000 or NextSeq500 sequencer (Illumina) using 695 custom sequencing primers with following read lengths: $50+10+12+50(\operatorname{Read} 1+$ 696 Index1 + Index2 + Read2). Primer and index sequences are listed in Supplementary 697 Table 8.

\section{Single nucleus RNA-seq data generation}

700 Droplet-based Chromium Single Cell 3' solution (10x Genomics, v3 chemistry)(Zheng 701 et al., 2017) was used to generate snRNA-seq libraries. Briefly, $30 \mathrm{mg}$ pulverized lung 702 tissue was resuspended in $500 \mu$ of nuclei permeabilization buffer $(0.1 \%$ Triton $\mathrm{X}-100$ 703 (Sigma-Aldrich, T8787), 1X protease inhibitor, $1 \mathrm{mM} \mathrm{DTT}$, and $0.2 \mathrm{U} / \mu \mathrm{l}$ RNase inhibitor 704 (Promega, N211B), 2\% BSA (Sigma-Aldrich, SRE0036) in PBS). Sample was incubated 705 on a rotator for 5 minutes at $4^{\circ} \mathrm{C}$ and then centrifuged at 500 rcf for 5 minutes $\left(4^{\circ} \mathrm{C}\right.$, run 706 speed 3/3). Supernatant was removed and pellet was resuspended in $400 \mu$ l of sort buffer 
707 (1 mM EDTA $0.2 \mathrm{U} / \mu \mathrm{l}$ RNase inhibitor (Promega, N211B), 2\% BSA (Sigma-Aldrich, 708 SRE0036) in PBS) and stained with DRAQ7 (1:100; Cell Signaling, 7406). 75,000 nuclei 709 were sorted using a SH800 sorter (Sony) into $50 \mu$ l of collection buffer consisting of $1 \mathrm{U} / \mu \mathrm{l}$ 710 RNase inhibitor in 5\% BSA; the FACS gating strategy sorted based on particle size and 711 DRAQ7 fluorescence. Sorted nuclei were then centrifuged at $1000 \mathrm{rcf}$ for 15 minutes $\left(4^{\circ} \mathrm{C}\right.$, 712 run speed 3/3) and supernatant was removed. Nuclei were resuspended in $35 \mu$ of 713 reaction buffer (0.2 U/ $\mu$ l RNase inhibitor (Promega, N211B), 2\% BSA (Sigma-Aldrich, 714 SRE0036) in PBS) and counted on a hemocytometer. 12,000 nuclei were loaded onto a 715 Chromium controller (10x Genomics). Libraries were generated using the Chromium 716 Single Cell 3' Library Construction Kit v3 (10x Genomics, 1000078) according to 717 manufacturer specifications. CDNA was amplified for 12 PCR cycles. SPRISelect reagent 718 (Beckman Coulter) was sued for size selection and clean-up steps. Final library 719 concentration was assessed by Qubit dsDNA HS Assay Kit (Thermo-Fischer Scientific) 720 and fragment size was checked using Tapestation High Sensitivity D1000 (Agilent) to 721 ensure that fragment sizes were distributed normally about 500 bp. Libraries were 722 sequenced using the NextSeq500 and a HiSeq4000 (Illumina) with these read lengths: $72328+8+91($ Read1 + Index1 + Read2).

\section{Single nucleus RNA-seq analysis}

726 Sequencing reads were demultiplexed (cellranger mkfastq) and processed (cellranger 727 count) using the Cell Ranger software package v3.0.2 (10x Genomics). Reads were 728 aligned to the human reference hg38 (Cell Ranger software package v3.0.2). Reads 729 mapping to intronic and exon sequences were retained. Resulting UMI feature-barcode 730 count matrices were loaded into Seurat (Stuart et al., 2019). All genes represented in >=3 731 nuclei and cells with 500-4000 detected genes were included for downstream processing. 732 UMI counts were log-normalized and scaled by a factor of 10,000 using the 733 NormalizeData function. Top 3000 variable features were identified using the 734 FindVariableFeatures function and finally scaled using the ScaleData function. Barcode 735 collisions were removed for individual datasets using DoubletFinder (McGinnis et al., 736 2019) with following parameters: $\mathrm{pN}=0.15$ and $\mathrm{pK}=0.005$, anticipated collision rate $=$

737 10\%. Clusters were assigned a doublet score (pANN) and classification as "doublet" or 
738 "singlet"; called doublets and cells with a pANN score > 0 were removed. UMI matrices

739 for datasets were merged and corrected for batch effects due to experiment date, donor,

740 and sex using the Harmony package (Korsunsky et al., 2019). UMAP coordinates and

741 clustering were performed using the RunUMAP, FindNeighbors, and FindClusters

742 functions in Seurat with principal components 1-23. 25-26, and 28. Clusters were

743 annotated, and putative doublets as defined by expression of canonically mutually

744 exclusive markers were excluded from analysis; remaining cells were re-clustered using

745 the previously described parameters. Final cluster annotation was done using canonical

746 markers. For genes of interest such as (e.g. ACE2, TMPRSS2), nuclei with at least one

747 UMI for the gene were considered "expressing". To analyze changes in percentage of

748 nuclei expressing we performed One-way ANOVA (ANalysis Of VAriance) with post-hoc

749 Tukey HSD (Honestly Significant Difference) using GraphPad Prism version 8.0.0 for

750 Windows, GraphPad Software, San Diego, California USA, www.graphpad.com. Due to

751 one potential outlier in the 30wkGA group (D062) we performed in addition a simple t-test

752 comparing $3 \mathrm{yr}$ to $30 \mathrm{yr}$ groups. Differential gene expression analysis between $A C E 2^{+}$

753 and ACE2- AT2 cells we used FindAllMarkers with parameters logfc $=0$, min.pct $=0$,

754 test.use = "wilcox", verbose = TRUE.

Normalization and comparison of gene expression frequency across snRNA-seq and scRNA-seq datasets

758 Single cell RNA-seq (10x Genomics 3' v2) of 4 aged (>55yr) control lungs were 759 obtained from publicly available data (Morse et al., 2019; Reyfman et al., 2019). Raw 760 gene expression matrices were downloaded from Gene Expression Omnibus (GEO) 761 repository (GSE128033 and GSE122960). Cells were filtered using the following 762 commonly used criteria: $>500$ expressed genes and $<10 \%$ UMls mapped to mitochondrial 763 DNAs. In addition, cells with greater than or equal to 40,000 UMIs were excluded from 764 the downstream analysis; this filtration criterion was selected based on the distribution of 765 UMls in single cells in individual donors. Seurat (version 3) (Stuart et al., 2019) was used 766 to identify AT2 cells from individual aged donors. Nuclei from the 9 libraries generated in 767 this study and cells from libraries for the 4 aged donors were integrated using the Seurat 7683 standard integration pipeline (Stuart et al., 2019). 
We calculated ACE2 and TMPRSS2 expression frequency in AT2 cells (percentage of AT2 cells with $>0 \mathrm{UMI}$ ) in individual donors. We then performed median based

771 normalization, so all donors reached the same median value. In calculating the median

772 value for each donor, the expression frequency values of genes $(n=26,260)$ common in

773 both datasets were used.

\section{Single nucleus ATAC-seq analysis}

776 For each sequenced snATAC-Seq libraries, we obtained four FASTQ files paired-end 777 DNA reads as well as the combinatorial indexes for i5 (768 different PCR indices) and T7

778 (96 different tagmentation indices; Supplementary Table 8). We selected all reads with $779<=2$ mistakes per individual index (Hamming distance between each pair of indices is 4) 780 and subsequently integrated the full barcode at the beginning of the read name in the 781 FASTQ files (https://gitlab.com/Grouumf/ATACdemultiplex/). Next, we used trim galore 782 (v.0.4.4) to remove adapter sequences from reads prior to read alignment. We aligned 783 reads to the hg19 reference genome using bwa mem (v.0.7.17) (Li and Durbin, 2009) and 784 subsequently used samtools ( $\mathrm{Li}$ et al., 2009) to remove unmapped, low map quality 785 (MAPQ<30), secondary, and mitochondrial reads. We then removed duplicate reads on 786 a per-cell basis using MarkDuplicates (BARCODE_TAG) from the picard toolkit. As an 787 initial quality cutoff, we set a minimum of 1,000 reads (unique, non-mitochondrial) and 788 observed 120,090 cells passing this threshold.

789 We used a previously described pipeline to identify snATAC-seq clusters (Chiou et 790 al., 2019). Briefly, we used scanpy (Wolf et al., 2018) to uniform read depth-normalize 791 and log-transform read counts within $5 \mathrm{~kb}$ windows. We then identified highly variable (hv) 792 windows (min_mean=0.01, min_disp=0.25) and regressed out the total read depth across $793 h v$ windows (usable counts) within each experiment. We then merged cells across 794 experiments and extracted the top 50 PCs, using Harmony (Korsunsky et al., 2019) to 795 correct for potential confounding factors including donor-of-origin and biological sex. We 796 used Harmony-corrected components to build a nearest neighbor graph 797 (n_neighbors=30) using the cosine metric, which was used for UMAP visualization 798 (min_dist=0.3) and Leiden clustering (resolution=1.5) (Traag et al., 2019). 
Prior to the final clustering results, we performed iterative clustering to identify and remove cells mapping to clusters with aberrant quality metrics. First, we removed 3,183 cells mapping in clusters with low read depth. Next, we removed 20,718 cells mapping in clusters with low fraction of reads in peaks. Finally, we re-clustered the cells at high resolution and removed 5,209 cells mapping in potential doublet sub-clusters. On average, these sub-clusters had higher usable counts, promoter usage, and accessibility at more than one marker gene promoter. After removing all of these cells, our final clusters consisted of 90,980 cells. To identify marker genes for each cluster, we used linear regression models with gene accessibility as a function of cluster assignment and usable counts across single cells.

\section{Computing relative accessibility scores}

We define an accessible locus as the minimal genomic region that can be bound and

812 cut by the enzyme. We use $L \subset N$ to represent the set of all accessible loci. We further

813 define a pseudo-locus as the set of accessible loci that relates to each other in a certain

814 meaningful way (for example, nearby loci, loci from different alleles). In this example,

815 pseudo-loci correspond to peaks. We use $\left\{d_{i} \mid d_{i} \subset L\right\}$ to represent the set of all pseudo-

816 loci. Let $a_{l}$ be the accessibility of accessible locus $l$, where $l \in L$. We define the 817 accessibility of pseudo-locus $d_{i}$ as $A_{i}=\sum_{k \in d_{i}} a_{k}$, i.e., the sum of accessibility of 818 accessible loci associated with di. Let $C_{j}$ be the library complexity (the number of distinct 819 molecules in the library) of cell $j$. Assuming unbiased PCR amplification, then the

820 probability of being sequenced for any fragment in the library is: $s_{j}=1-\left(1-\frac{1}{C_{j}}\right) k_{j}$, where

$821 k_{j}$ is the total number of reads for cell $j$. If we assume that the probability of a fragment

822 present in the library is proportional to its accessibility and the complexity of the library,

823 then we can deduce that the probability of a given locus $l$ in cell $j$ being sequenced is:

$824 p_{l j} \propto a_{l} C_{j} s_{j}$. For any pseudo-locus $d_{i}$, the number of reads in $d_{i}$ for cell $j$ follows the 825 Poisson binomial distribution, and its mean is $m_{i j}=\sum_{k \in d_{i}} p_{k j} \propto C_{j} s_{j} \sum_{k \in d_{i}} a_{k}=C_{j} s_{j} A_{i}$.

826 Given a pseudo-locus (or peak) by cell count matrix $O$, we have: $\sum_{j} O_{i j}=\sum_{j} m_{i j}$.

827 Therefore, $A_{i}=Z \frac{\sum_{j} o_{i j}}{\sum_{j} C_{j} s_{j}}$, where $Z$ is a normalization constant. When comparing across 
828 different samples the relative accessibility may be desirable as they sum up to a constant,

829 i.e., $\sum_{i} A_{i}=1 \times 10^{6}$. In this case, we can derive $A_{i}=\frac{\sum_{j} o_{i j}}{\sum_{i j} o_{i j}} * 10^{6}$.

831 Calculating the relative percent of cells with accessibility at a locus

832 To correct for biases occurring from differential read depths between clusters, we used

833 the following strategy to determine the relative ratio of cells with accessibility at a given

834 locus. We defined the set of accessible loci $L$ of a given dataset $D$ as the genomic regions

835 covered by the set peaks $P$ inferred from $D$. We define $X$ the set of cells from $D$, and $S$ a 836 partitioning of $X$. For a given partition $S_{i} \in S$ and for each feature $p_{j} \in P$, we computed

$837 m_{i j}$ the ratio of cells from $S_{i}$ with at least one read overlapping $p_{j}$. We then defined the

838 score $s_{i j}$ of loci $p_{j}$ in $S_{i}$ as $s_{i j}=10^{6} \cdot \frac{m_{i j}}{\sum_{j \in P} m_{i j}}$. We finally define the relative ratio of cells

839 normalized across the different clusters as $R S_{i j}=\frac{s_{i j}}{\sum_{i \in S} s_{i j}}$.

Associating promoters to candidate distal regulatory elements.

842 To identify AT2 co-accessible loci with the promoters of TMPRSS2, ACE2, FURIN, 843 BSG, and CTSL we used Cicero (Pliner et al., 2018). First, we performed a Cicero 844 analysis for each individual cluster using a genomic window of $1 \mathrm{Mb}$ (co-accessibility 845 score >0.05). In addition, we performed Cicero using a random subset of 15,000 nuclei 846 from the complete dataset and a genomic window of $250 \mathrm{~kb}$ (co-accessibility score >0.05).

847 We then defined the promoter regions of ACE2, TMPRSS2, FURIN, BSG, and CTSL as 848 transcriptional start site (TSS) +/- $1 \mathrm{~kb}$ and selected the sites co-accessible with each of 849 the promoters (co-accessibility score >0.05). Finally, we merged the elements co850 accessible with the gene promoters from both analyses to generate a union set of 851 candidate elements.

853 Identification and clustering of AT2 peaks with changes in chromatin accessibility 854 genome-wide

855 We used edgeR (Robinson et al., 2010) to identify differential accessible peaks 856 between each of pair of time points. As input we used the 122,352 peaks in AT2 cell. 
857 Dataset ID and sex were used as technical covariates. Sites with False Discovery Rate $858($ FDR $)<0.05$ after Benjamini-Hochberg correction were considered significant. Next, we 859 performed K-means using the relative accessibility score with a loci $x$ timepoints matrix.

860 We used $\mathrm{K}$ from 5 to 8 and computed the Davis-Bouldin index to determine the best $\mathrm{K}$ to

861 partition the loci. let $R_{x y}=\frac{\left(s_{x}+s_{y}\right)}{d_{x y}}$ with $s_{x}$ the average distance of each sample from cluster

$862 x$ and $d_{x y}$ the distance between the centroids of clusters $x$ and $y$. The Davies-Bouldin

863 index is defined as $D B=\frac{1}{K} \sum_{x, y \in} \max _{x \neq y}\left(R_{x y}\right)$ and low $D B$ scores indicate better partitioning.

864 We obtained an optimal partition with $\mathrm{K}=5$.

Identification of AT2 peaks with changes in chromatin accessibility at candidate gene loci

The ensemble of cells $X$ from $D$ can be divided per timepoint, cell subtype, or donor. We identified for individual donors the relative \% of cells with at least one read in peaks associated with ACE2, TMPRSS2, FURIN, BSG, and CTSL promoters. As a background to calculate the relative \% of cells, we used the merged set of peaks from all the clusters. Then, we computed a Student test for two independent samples with equal variance for each pair of categories: $30 w^{G A}, 3$ yo and 30 yo. For each element the relative $\%$ of cells

Annotation of genomic elements were used as measurement variable and the timepoint as nominal variable.

The GREAT algorithm (McLean et al., 2010) was used to annotate distal genomic

\section{Transcription factor related analyses}

881 De novo motif enrichment analysis in genomic elements was performed using 882 HOMER (Heinz et al., 2010) with standard parameters. Motif scanning was performed 883 using FIMO (Grant et al., 2011) online interface and default parameters. Motif files were 884 downloaded from JASPAR (Fornes et al., 2020) in MEME format. Motifs scanned were 885 MA0102.4 (CEBPA), MA0673.1(NKX2-8), MA0153.1(HNF1B), MA0503.1(NKX2-5), MA0877.2(BARHL1), PB0022.1(GATA5), MA0490.1(JUNB), PH0171.1(NKX2-1), 
MA0148.1(FOXA1), MA0144.1(STAT3), MA0517.1(STAT1::STAT2), MA0050.1(IRF),

888 MA0007.2(AR), and MA0592.1(ESRRA). To identify overlap with TF ChIP-seq sites, we 889 used ChIP-atlas (Oki et al., 2018). We downloaded a BED file for "TFs and other" antigens

890 across all lung related samples from the Peak Browser. We intersected these peaks with 891 the TMPRSS2-linked peaks and the FIMO motifs (Grant et al., 2011). In addition, we 892 downloaded enhancer related histone modifications (H3K4me1, H3K27ac) from the 893 SCREEN database and intersected with the peak lists (Consortium, 2012).

\section{Predicting variant effects on chromatin accessibility}

896 We used deltaSVM (Lee et al., 2015) to predict the effects of variants on chromatin 897 accessibility in AT2 cells. First, we extracted the sequences underlying AT2 sites that were promoter-distal (> \pm 500 bp from GENCODE v19 transcript TSS for protein-coding and long non-coding RNA genes). As described previously (Chiou et al., 2019), we trained

900 an AT2 sequence-based model and used it to predict effects for all possible combinations 901 of 11 mers. Next, to compile a comprehensive set of variants to test, we downloaded lists 902 of variants from gnomAD v3 (Karczewski et al., 2019) and filtered out variants that were 903 singletons or indels longer than $3 \mathrm{bp}$. We then used the liftOver (Tyner et al., 2017) utility 904 to transform GRCh38 into GRCh37/hg19 coordinates. We retained variants from either 905 dataset that mapped within TMPRSS2 linked sites and extracted sequences in a $19 \mathrm{bp}$ 906 window around each variant ( \pm 9 bp flanking each side). Finally, we calculated deltaSVM 907 z-scores for each variant by predicting deltaSVM scores, randomly permuting 11 mer 908 effects and re-predicting deltaSVM scores, and using the parameters of the null 909 distribution to calculate deltaSVM z-scores. From the z-scores, we calculated p-values 910 and q-values and defined variants with significant effects using a threshold of FDR<0.1.

911 We identified common variants defined as minor allele frequency >.01 in at least one 912 major population group. For each common variant, we obtained sequence surrounding 913 each variant allele and predicted sequence motifs from the JASPAR database (Fornes et 914 al., 2020) using FIMO (Grant et al., 2011), and focused on motifs of TF families enriched 915 in age-dependent AT2 chromatin.

916

917 Phenotype associations for predicted effect variants 
We downloaded UK biobank round 2 GWAS combined sex results (Lab, 2020; Sudlow

919 et al., 2015). We used broad disease categories from the ICD-10-CM to classify ICD10

920 phenotypes, except for ICD10 codes relating to unclassified symptoms, external causes

921 of morbidity, and factors influencing health status and contact with health services. We

922 combined all non-cancer, self-reported diseases into a single category (self-reported) as

923 well as all treatments and medications (medication). We then extracted GWAS

924 association results for variants that were not tagged as low confidence variants, had

925 significant deltaSVM effects, and mapped in TMPRSS2-linked aging-related sites. From

926 these variants, we removed one (rs199938061) which was in perfect linkage

927 disequilibrium with another variant.

\section{Deconvoluting the TMPRSS2 lung eQTL}

930 We used MuSiC (v.0.1.1) (Wang et al., 2019) to estimate the proportions of lung cell

931 types with >500 cells from our scRNA-seq dataset in lung bulk RNA-seq samples from

932 the GTEx v8 release (Aguet et al., 2019). We combined cell type labels for capillary (distal

933 and proximal), macrophages (M1 and M2), matrix fibroblasts (1 and 2), and NK/T cells.

934 We modeled the relationship between TMM-normalized TMPRSS2 expression as a

935 function of the interaction between genotype and cell type proportion, while considering

936 the covariates used in the original GTEx data including sex, sequencing platform, PCR,

9375 genotype PCs, and 59 inferred PCs from the expression data. From the original inferred

938 PCs, we excluded inferred PC 1 because it was highly correlated with AT2 cell type

939 proportion (Spearman $\rho=0.67$ ). 
943 Figure S1. Quality control of snRNA-seq and snATAC-seq datasets. A

944 Representative UMI barcode distribution output from CellRanger pipeline for snRNA-seq

945 libraries from human lung. B Number of nuclei passing quality control filtering for snRNA-

946 seq libraries. C Genes detected per nucleus. D Sequencing saturation of snRNA-seq

947 libraries. E Nuclei with less than 1,000 uniquely mapped reads were filtered from 948 snATAC-seq datasets. F Number of nuclei passing quality control filtering for snATAC949 seq libraries. G Average number of reads per nucleus. H Fraction of reads in peak regions 950 per dataset. All data are represented as mean \pm SD.

Figure S2. Marker plots for cluster annotation and expression profiling of candidate

953 genes involved in SARS-CoV-2 cell entry. A Dot plot of marker genes used for cluster

954 annotation. B-D Cell type specific gene expression of candidate genes for cell entry. Violin

955 plots display expression values per nucleus for genes encoding B Cathepsin L (CTSL),

956 C FURIN (FURIN) and D Basigin (BSG, CD147). E Correlation of ACE2+ and TMPRSS2+

957 AT2 cells with linear regression. F, G Fraction of AT2 cells with expression of ACE2 and

958 TMPRSS2 at each time point. Data are the same as Fig. 1D, E, but with potential outlier

959 sample D062 removed. * $p<0.05$ (One-way ANOVA with post-hoc Tukey test).

Figure S3. Expression analysis of viral entry genes. Displayed are violin plots of expression levels for entry genes related to other viruses including SARS-CoV, MERS,

963 coronavirus associated with common cold, Rhinovirus, Respiratory Syncytial Virus (RSV), 964 Adenovirus, Influenza Virus.

Figure S4. Integrative analysis of ACE2 and TMPRSS2 expression in lungs from

967 aged individuals. A Seurat3 Standard Integration (Stuart et al., 2019) was applied to 968 snRNA-seq data for 9 donors generated as part of this study and publicly available 969 scRNA-seq datasets 4 additional donor lungs (age> 55). AT2 cells from 13 donors were 970 clustered together via Louvain clustering with minimal batch variation. Left panel: t-SNE 971 visualization of cells colored by major cell type annotation. Epi other: predicted non-AT2 
972 epithelial cells. Right panel: t-SNE visualization of cells colored by donor information. B

973 Normalized expression frequency of ACE2 (left) and TMPRSS2 (right) in AT2 cells. p

974 value was calculated using one-tailed t-test comparing normalized frequency in donors of

975 30yo group and aged group.

976

977 Figure S5. Marker plots for cluster annotation of snATAC-seq and profiling of peaks

978 at candidate genes for SARS-CoV-2 cell entry. A Dot plot of marker genes used for

979 cluster annotation. B-D Cell type resolved chromatin accessibility at peaks within +/- 650

$980 \mathrm{~kb}$ of candidate genes for cell entry. Displayed are data for B FURIN (FURIN) and C

981 Basigin (BSG, CD147) D Cathepsin L (CTSL). Values are displayed as row normalized

982 proportion of cells with a fragment in a peak region. Black asterisks denote co-

983 accessibility from Cicero >0.05 (Cusanovich et al., 2018).

985 Figure S6. Quantification of peaks with increased accessibility with age at tested 986 loci and donor resolved activity of sites not increased at TMPRSS2 locus. A Number

987 of peaks within +/- $650 \mathrm{~kb}$ of candidate genes for cell entry overlapping clll and clV from

988 Figure 3B. B Number of peaks co-accessible with the promoter of candidate genes for 989 cell entry overlapping clll and clV from Figure 3B. C Donor resolved analysis of 24/37 990 peaks at the TMPRSS2 gene locus. Red asterisks denote FDR <0.05 (EdgeR) and black 991 asterisks denote $p<0.05$ via t-test. 
995

\section{SUPPLEMENTARY TABLE LEGENDS}

Supplementary Table 1. Donor metadata tables. Sheet 1: $30 \mathrm{wk}^{\mathrm{GA}}$ - 30yo: Donor ID, age, sex, race, clinical pathology diagnosis (clinPathDx), gestational age, overall quality of the lung tissue assessment, type of death and cause of death were listed. Not shown are data on body weight, body height, total lung weight and radial alveolar count assessment of alveolarization. All were all within normal limits for age. Abbreviations: DCD: donor after cardiac death; DBD: donor after brain death; GA: gestational age; RDS: respiratory distress syndrome. Sheet 2: aged cohort: Donor ID, age, sex, smoking history, race and cause of death were listed (Morse et al., 2019; Reyfman et al., 2019).

Supplementary Table 2. Cluster composition and number and fraction of nuclei expressing candidate for SARS-CoV2 cell entry.

Supplementary Table 3. Differential expressed analysis between $A C E 2^{+}$and $A C E 2^{-}$as well as TMPRSS2 ${ }^{+}$and TMPRSS2- AT2 cells.

Supplementary Table 4. Annotation of peaks within a window of $+/-650 \mathrm{~kb}$ of candidate genes for SARS-CoV2 cell entry.

Supplementary Table 5. Annotation of peaks co-accessible with candidate genes for SARS-CoV2 cell entry and age-associated changes of chromatin accessibility of peaks co-accessible with TMPRSS2 promoter.

Supplementary Table 6. GREAT analysis of peaks increasing with age in AT2 cells (groups clll and clV in Fig 3B).

Supplementary Table 7. De novo motif enrichment analysis of peaks increasing with age in AT2 cells (groups clll and clV in Fig 3B).

Supplementary Table 8. Indexes and primer sequences for snATAC-seq libraries. 


\section{REFERENCES}

1026

1027

1028

1029

1030

1031

1032

1033

1034

1035

1036

1037

1038

1039

1040

1041

1042

1043

1044

1045

1046

1047

1048

1049

1050

1051

1052

1053

1054

1055

1056

1057

1058

1059

1060

1061

1062

1063

1064

1065

1066

1067

1068

1069

Aguet, F., Barbeira, A.N., Bonazzola, R., Brown, A., Castel, S.E., Jo, B., Kasela, S., KimHellmuth, S., Liang, Y., Oliva, M., et al. (2019). The GTEx Consortium atlas of genetic regulatory effects across human tissues. bioRxiv.

Ardini-Poleske, M.E., Clark, R.F., Ansong, C., Carson, J.P., Corley, R.A., Deutsch, G.H., Hagood, J.S., Kaminski, N., Mariani, T.J., Potter, S.S., et al. (2017). LungMAP: The Molecular Atlas of Lung Development Program. Am J Physiol Lung Cell Mol Physiol 313, L733-L740.

Bandyopadhyay, G., Huyck, H.L., Misra, R.S., Bhattacharya, S., Wang, Q., Mereness, J., Lillis, J., Myers, J.R., Ashton, J., Bushnell, T., et al. (2018). Dissociation, cellular isolation, and initial molecular characterization of neonatal and pediatric human lung tissues. Am J Physiol Lung Cell Mol Physiol 315, L576-L583.

Battles, M.B., and McLellan, J.S. (2019). Respiratory syncytial virus entry and how to block it. Nat Rev Microbiol 17, 233-245.

Bi, Q., Wu, Y., Mei, S., Ye, C., Zou, X., Zhang, Z., Liu, X., Wei, L., Truelove, S.A., Zhang, T., et al. (2020). Epidemiology and Transmission of COVID-19 in Shenzhen China: Analysis of 391 cases and 1,286 of their close contacts. medRxiv.

Bochkov, Y.A., and Gern, J.E. (2016). Rhinoviruses and Their Receptors: Implications for Allergic Disease. Curr Allergy Asthma Rep 16, 30.

Buenrostro, J.D., Giresi, P.G., Zaba, L.C., Chang, H.Y., and Greenleaf, W.J. (2013). Transposition of native chromatin for fast and sensitive epigenomic profiling of open chromatin, DNA-binding proteins and nucleosome position. Nat Methods 10, 12131218.

Buenrostro, J.D., Wu, B., Litzenburger, U.M., Ruff, D., Gonzales, M.L., Snyder, M.P., Chang, H.Y., and Greenleaf, W.J. (2015). Single-cell chromatin accessibility reveals principles of regulatory variation. Nature 523, 486-490.

CDC, C.-R.T. (2020). Coronavirus Disease 2019 in Children - United States, February 12-April 2, 2020. MMWR Morb Mortal Wkly Rep 2020 69, 422-426.

Chen, X., Miragaia, R.J., Natarajan, K.N., and Teichmann, S.A. (2018). A rapid and robust method for single cell chromatin accessibility profiling. Nat Commun 9, 5345.

Chen, Z., Mi, L., Xu, J., Yu, J., Wang, X., Jiang, J., Xing, J., Shang, P., Qian, A., Li, Y., et al. (2005). Function of HAb18G/CD147 in invasion of host cells by severe acute respiratory syndrome coronavirus. J Infect Dis 191, 755-760.

Chiou, J., Zeng, C., Cheng, Z., Han, J.Y., Schlichting, M., Huang, S., Wang, J., Sui, Y., Deogaygay, A., Okino, M.-L., et al. (2019). Single cell chromatin accessibility reveals pancreatic islet cell type- and state-specific regulatory programs of diabetes risk. bioRxiv.

Consortium, E.P. (2012). An integrated encyclopedia of DNA elements in the human genome. Nature 489, 57-74.

Corces, M.R., Shcherbina, A., Kundu, S., Gloudemans, M.J., Frésard, L., Granja, J.M., Louie, B.H., Shams, S., Bagdatli, S.T., Mumbach, M.R., et al. (2020). Single-cell epigenomic identification of inherited risk loci in Alzheimer's and Parkinson's disease. bioRxiv.

Corces, M.R., Trevino, A.E., Hamilton, E.G., Greenside, P.G., Sinnott-Armstrong, N.A., Vesuna, S., Satpathy, A.T., Rubin, A.J., Montine, K.S., Wu, B., et al. (2017). An 
improved ATAC-seq protocol reduces background and enables interrogation of frozen tissues. Nat Methods 14, 959-962.

Coutard, B., Valle, C., de Lamballerie, X., Canard, B., Seidah, N.G., and Decroly, E. (2020). The spike glycoprotein of the new coronavirus 2019-nCoV contains a furinlike cleavage site absent in CoV of the same clade. Antiviral Res 176, 104742. Steemers, F.J., Trapnell, C., and Shendure, J. (2015). Multiplex single cell profiling of chromatin accessibility by combinatorial cellular indexing. Science 348, 910-914.

1077

1078

1079

Cusanovich, D.A., Hill, A.J., Aghamirzaie, D., Daza, R.M., Pliner, H.A., Berletch, J.B., Filippova, G.N., Huang, X., Christiansen, L., DeWitt, W.S., et al. (2018). A Single-Cell Atlas of In Vivo Mammalian Chromatin Accessibility. Cell 174, 1309-1324 e1318. al. (2020). Clinical Features of 85 Fatal Cases of COVID-19 from Wuhan: A Retrospective Observational Study. Am J Respir Crit Care Med.

1083

1084

1085

Fang, R., Preissl, S., Hou, X., Lucero, J., Wang, X., Motamedi, A., Shiau, A.K., Mukamel, E.A., Zhang, Y., Behrens, M.M., et al. (2019). Fast and Accurate Clustering of Single

1086

1087 Cell Epigenomes Reveals <em $>$ Cis $</$ em>-Regulatory Elements in Rare Cell Types. bioRxiv.

1088

1089

Fornes, O., Castro-Mondragon, J.A., Khan, A., van der Lee, R., Zhang, X., Richmond, P.A., Modi, B.P., Correard, S., Gheorghe, M., Baranasic, D., et al. (2020). JASPAR 2020: update of the open-access database of transcription factor binding profiles. Nucleic Acids Res 48, D87-D92.

1093

1094

1095

1096

1097

1098

1099

Grant, C.E., Bailey, T.L., and Noble, W.S. (2011). FIMO: scanning for occurrences of a given motif. Bioinformatics 27, 1017-1018.

Heinz, S., Benner, C., Spann, N., Bertolino, E., Lin, Y.C., Laslo, P., Cheng, J.X., Murre, C., Singh, H., and Glass, C.K. (2010). Simple combinations of lineage-determining transcription factors prime cis-regulatory elements required for macrophage and $\mathrm{B}$ cell identities. Mol Cell 38, 576-589.

Huang, I.C., Bosch, B.J., Li, W., Farzan, M., Rottier, P.M., and Choe, H. (2006). SARSCoV, but not HCoV-NL63, utilizes cathepsins to infect cells: viral entry. Adv Exp Med Biol 581, 335-338.

Imai, Y., Kuba, K., Rao, S., Huan, Y., Guo, F., Guan, B., Yang, P., Sarao, R., Wada, T., Leong-Poi, H., et al. (2005). Angiotensin-converting enzyme 2 protects from severe acute lung failure. Nature 436, 112-116.

Iwata-Yoshikawa, N., Okamura, T., Shimizu, Y., Hasegawa, H., Takeda, M., and Nagata, N. (2019). TMPRSS2 Contributes to Virus Spread and Immunopathology in the Airways of Murine Models after Coronavirus Infection. J Virol 93.

Karczewski, K.J., Francioli, L.C., Tiao, G., Cummings, B.B., Alföldi, J., Wang, Q., Collins, R.L., Laricchia, K.M., Ganna, A., Birnbaum, D.P., et al. (2019). Variation across 141,456 human exomes and genomes reveals the spectrum of loss-of-function intolerance across human protein-coding genes. bioRxiv.

Kim, T.S., Heinlein, C., Hackman, R.C., and Nelson, P.S. (2006). Phenotypic analysis of mice lacking the Tmprss2-encoded protease. Mol Cell Biol 26, 965-975.

Korsunsky, I., Millard, N., Fan, J., Slowikowski, K., Zhang, F., Wei, K., Baglaenko, Y., Brenner, M., Loh, P.R., and Raychaudhuri, S. (2019). Fast, sensitive and accurate integration of single-cell data with Harmony. Nat Methods 16, 1289-1296. 
1116 Kuba, K., Imai, Y., Rao, S., Gao, H., Guo, F., Guan, B., Huan, Y., Yang, P., Zhang, Y., Deng, W., et al. (2005). A crucial role of angiotensin converting enzyme 2 (ACE2) in SARS coronavirus-induced lung injury. Nat Med 11, 875-879. L., et al. (2020). Structure of the SARS-CoV-2 spike receptor-binding domain bound to the ACE2 receptor. Nature.

Laporte, M., and Naesens, L. (2017). Airway proteases: an emerging drug target for influenza and other respiratory virus infections. Curr Opin Virol 24, 16-24.

Lareau, C.A., Duarte, F.M., Chew, J.G., Kartha, V.K., Burkett, Z.D., Kohlway, A.S., Pokholok, D., Aryee, M.J., Steemers, F.J., Lebofsky, R., et al. (2019). Droplet-based combinatorial indexing for massive-scale single-cell chromatin accessibility. Nat Biotechnol 37, 916-924.

Lee, D., Gorkin, D.U., Baker, M., Strober, B.J., Asoni, A.L., McCallion, A.S., and Beer, M.A. (2015). A method to predict the impact of regulatory variants from DNA sequence. Nat Genet 47, 955-961.

$\mathrm{Li}, \mathrm{H}$., and Durbin, R. (2009). Fast and accurate short read alignment with BurrowsWheeler transform. Bioinformatics 25, 1754-1760.

Li, H., Handsaker, B., Wysoker, A., Fennell, T., Ruan, J., Homer, N., Marth, G., Abecasis, G., Durbin, R., and Genome Project Data Processing, S. (2009). The Sequence Alignment/Map format and SAMtools. Bioinformatics 25, 2078-2079.

Limburg, H., Harbig, A., Bestle, D., Stein, D.A., Moulton, H.M., Jaeger, J., Janga, H., Hardes, K., Koepke, J., Schulte, L., et al. (2019). TMPRSS2 Is the Major Activating Protease of Influenza A Virus in Primary Human Airway Cells and Influenza B Virus in Human Type II Pneumocytes. J Virol 93.

Lukhele, S., Boukhaled, G.M., and Brooks, D.G. (2019). Type I interferon signaling, regulation and gene stimulation in chronic virus infection. Semin Immunol 43, 101277.

Matsuyama, S., Nao, N., Shirato, K., Kawase, M., Saito, S., Takayama, I., Nagata, N., Sekizuka, T., Katoh, H., Kato, F., et al. (2020). Enhanced isolation of SARS-CoV-2 by TMPRSS2-expressing cells. Proc Natl Acad Sci U S A 117, 7001-7003.

Maurano, M.T., Haugen, E., Sandstrom, R., Vierstra, J., Shafer, A., Kaul, R., and Stamatoyannopoulos, J.A. (2015). Large-scale identification of sequence variants influencing human transcription factor occupancy in vivo. Nat Genet 47, 1393-1401.

McGinnis, C.S., Murrow, L.M., and Gartner, Z.J. (2019). DoubletFinder: Doublet Detection in Single-Cell RNA Sequencing Data Using Artificial Nearest Neighbors. Cell Syst 8, 329-337 e324.

McLean, C.Y., Bristor, D., Hiller, M., Clarke, S.L., Schaar, B.T., Lowe, C.B., Wenger, A.M., and Bejerano, G. (2010). GREAT improves functional interpretation of cis-regulatory regions. Nat Biotechnol 28, 495-501.

Mesev, E.V., LeDesma, R.A., and Ploss, A. (2019). Decoding type I and III interferon signalling during viral infection. Nat Microbiol 4, 914-924.

Morse, C., Tabib, T., Sembrat, J., Buschur, K.L., Bittar, H.T., Valenzi, E., Jiang, Y., Kass, D.J., Gibson, K., Chen, W., et al. (2019). Proliferating SPP1/MERTK-expressing macrophages in idiopathic pulmonary fibrosis. Eur Respir $\mathrm{J} 54$.

Narayanan, M., Owers-Bradley, J., Beardsmore, C.S., Mada, M., Ball, I., Garipov, R., Panesar, K.S., Kuehni, C.E., Spycher, B.D., Williams, S.E., et al. (2012). 
Alveolarization continues during childhood and adolescence: new evidence from helium-3 magnetic resonance. Am J Respir Crit Care Med 185, 186-191.

Ochs, M., Nyengaard, J.R., Jung, A., Knudsen, L., Voigt, M., Wahlers, T., Richter, J., and Gundersen, H.J. (2004). The number of alveoli in the human lung. Am J Respir Crit Care Med 169, 120-124.

Oki, S., Ohta, T., Shioi, G., Hatanaka, H., Ogasawara, O., Okuda, Y., Kawaji, H., Nakaki, R., Sese, J., and Meno, C. (2018). ChIP-Atlas: a data-mining suite powered by full integration of public ChIP-seq data. EMBO Rep 19.

Peck, K.M., Burch, C.L., Heise, M.T., and Baric, R.S. (2015). Coronavirus Host Range Expansion and Middle East Respiratory Syndrome Coronavirus Emergence: Biochemical Mechanisms and Evolutionary Perspectives. Annu Rev Virol 2, 95-117.

Pickrell, J.K. (2014). Joint analysis of functional genomic data and genome-wide association studies of 18 human traits. Am J Hum Genet 94, 559-573.

Pliner, H.A., Packer, J.S., McFaline-Figueroa, J.L., Cusanovich, D.A., Daza, R.M., Aghamirzaie, D., Srivatsan, S., Qiu, X., Jackson, D., Minkina, A., et al. (2018). Cicero Predicts cis-Regulatory DNA Interactions from Single-Cell Chromatin Accessibility Data. Mol Cell 71, 858-871 e858.

Preissl, S., Fang, R., Huang, H., Zhao, Y., Raviram, R., Gorkin, D.U., Zhang, Y., Sos, B.C., Afzal, V., Dickel, D.E., et al. (2018). Single-nucleus analysis of accessible chromatin in developing mouse forebrain reveals cell-type-specific transcriptional regulation. Nat Neurosci 21, 432-439.

Qi, F., Qian, S., Zhang, S., and Zhang, Z. (2020). Single cell RNA sequencing of 13 human tissues identify cell types and receptors of human coronaviruses. bioRxiv.

Raj, V.S., Mou, H., Smits, S.L., Dekkers, D.H., Muller, M.A., Dijkman, R., Muth, D., Demmers, J.A., Zaki, A., Fouchier, R.A., et al. (2013). Dipeptidyl peptidase 4 is a functional receptor for the emerging human coronavirus-EMC. Nature 495, 251-254.

Reinke, L.M., Spiegel, M., Plegge, T., Hartleib, A., Nehlmeier, I., Gierer, S., Hoffmann, M., Hofmann-Winkler, H., Winkler, M., and Pöhlmann, S. (2017). Different residues in the SARS-CoV spike protein determine cleavage and activation by the host cell protease TMPRSS2. PLoS One 12, e0179177.

Reyfman, P.A., Walter, J.M., Joshi, N., Anekalla, K.R., McQuattie-Pimentel, A.C., Chiu, S., Fernandez, R., Akbarpour, M., Chen, C.I., Ren, Z., et al. (2019). Single-Cell Transcriptomic Analysis of Human Lung Provides Insights into the Pathobiology of Pulmonary Fibrosis. Am J Respir Crit Care Med 199, 1517-1536.

Roadmap Epigenomics, C., Kundaje, A., Meuleman, W., Ernst, J., Bilenky, M., Yen, A., Heravi-Moussavi, A., Kheradpour, P., Zhang, Z., Wang, J., et al. (2015). Integrative analysis of 111 reference human epigenomes. Nature 518, 317-330.

Robinson, M.D., McCarthy, D.J., and Smyth, G.K. (2010). edgeR: a Bioconductor package for differential expression analysis of digital gene expression data. Bioinformatics 26, 139-140.

Satpathy, A.T., Granja, J.M., Yost, K.E., Qi, Y., Meschi, F., McDermott, G.P., Olsen, B.N., Mumbach, M.R., Pierce, S.E., Corces, M.R., et al. (2019). Massively parallel singlecell chromatin landscapes of human immune cell development and intratumoral $\mathrm{T}$ cell exhaustion. Nat Biotechnol 37, 925-936.

Schiller, H.B., Montoro, D.T., Simon, L.M., Rawlins, E.L., Meyer, K.B., Strunz, M., Vieira Braga, F.A., Timens, W., Koppelman, G.H., Budinger, G.R.S., et al. (2019). The 
Human Lung Cell Atlas: A High-Resolution Reference Map of the Human Lung in Health and Disease. Am J Respir Cell Mol Biol 61, 31-41.

Stuart, T., Butler, A., Hoffman, P., Hafemeister, C., Papalexi, E., Mauck, W.M., 3rd, Hao, Y., Stoeckius, M., Smibert, P., and Satija, R. (2019). Comprehensive Integration of Single-Cell Data. Cell 177, 1888-1902 e1821.

1212

1213

1214

1215

1216

1217

1218

1219

1220

1221

1222

1223

1224

1225

1226

1227

1228

1229

1230

1231

1232

1233

1234

1235

1236

1237

1238

1239

1240

1241

1242

1243

1244

1245

1246

1247

1248

1249

1250

1251

Sudlow, C., Gallacher, J., Allen, N., Beral, V., Burton, P., Danesh, J., Downey, P., Elliott, P., Green, J., Landray, M., et al. (2015). UK biobank: an open access resource for identifying the causes of a wide range of complex diseases of middle and old age. PLoS Med 12, e1001779.

Thurman, R.E., Rynes, E., Humbert, R., Vierstra, J., Maurano, M.T., Haugen, E., Sheffield, N.C., Stergachis, A.B., Wang, H., Vernot, B., et al. (2012). The accessible chromatin landscape of the human genome. Nature 489, 75-82.

Traag, V.A., Waltman, L., and van Eck, N.J. (2019). From Louvain to Leiden: guaranteeing well-connected communities. Sci Rep 9, 5233.

Travaglini, K.J., Nabhan, A.N., Penland, L., Sinha, R., Gillich, A., Sit, R.V., Chang, S., Conley, S.D., Mori, Y., Seita, J., et al. (2020). A molecular cell atlas of the human lung from single cell RNA sequencing. bioRxiv.

Tyner, C., Barber, G.P., Casper, J., Clawson, H., Diekhans, M., Eisenhart, C., Fischer, C.M., Gibson, D., Gonzalez, J.N., Guruvadoo, L., et al. (2017). The UCSC Genome Browser database: 2017 update. Nucleic Acids Res 45, D626-D634.

Walls, A.C., Park, Y.J., Tortorici, M.A., Wall, A., McGuire, A.T., and Veesler, D. (2020). Structure, Function, and Antigenicity of the SARS-CoV-2 Spike Glycoprotein. Cell.

Wang, M., Wu, Q., Xu, W., Qiao, B., Wang, J., Zheng, H., Jiang, S., Mei, J., Wu, Z., Deng, Y., et al. (2020). Clinical diagnosis of 8274 samples with 2019-novel coronavirus in Wuhan. medRxiv.

Wang, X., Park, J., Susztak, K., Zhang, N.R., and Li, M. (2019). Bulk tissue cell type deconvolution with multi-subject single-cell expression reference. Nat Commun 10, 380 .

Waradon Sungnak, N.H., Christophe Bécavin, Marijn Berg, HCA Lung Biological Network (2020). SARS-CoV-2 Entry Genes Are Most Highly Expressed in Nasal Goblet and Ciliated Cells within Human Airways. arXiv.

Wolf, F.A., Angerer, P., and Theis, F.J. (2018). SCANPY: large-scale single-cell gene expression data analysis. Genome Biol 19, 15.

Wu, X., Cai, Y., Huang, X., Yu, X., Zhao, L., Wang, F., Li, Q., Gu, S., Xu, T., Li, Y., et al. (2020). Co-infection with SARS-CoV-2 and Influenza A Virus in Patient with Pneumonia, China. Emerg Infect Dis 26.

Xia, C., Anderson, P., and Hahm, B. (2018). Viral dedication to vigorous destruction of interferon receptors. Virology 522, 19-26.

Xu, Y., Mizuno, T., Sridharan, A., Du, Y., Guo, M., Tang, J., Wikenheiser-Brokamp, K.A., Perl, A.T., Funari, V.A., Gokey, J.J., et al. (2016). Single-cell RNA sequencing identifies diverse roles of epithelial cells in idiopathic pulmonary fibrosis. JCI Insight 1 , e90558.

Yan, R., Zhang, Y., Li, Y., Xia, L., Guo, Y., and Zhou, Q. (2020). Structural basis for the recognition of SARS-CoV-2 by full-length human ACE2. Science 367, 1444-1448. 
Yeager, C.L., Ashmun, R.A., Williams, R.K., Cardellichio, C.B., Shapiro, L.H., Look, A.T., and Holmes, K.V. (1992). Human aminopeptidase $\mathrm{N}$ is a receptor for human coronavirus 229E. Nature 357, 420-422. C., Myers, R.M., Brown, M., Li, W., et al. (2008). Model-based analysis of ChIP-Seq (MACS). Genome Biol 9, R137.

Zhao, Y., Zhao, Z., Wang, Y., Zhou, Y., Ma, Y., and Zuo, W. (2020). Single-cell RNA expression profiling of ACE2,thereceptor of SARS-CoV-2. bioRxiv.

Zheng, G.X., Terry, J.M., Belgrader, P., Ryvkin, P., Bent, Z.W., Wilson, R., Ziraldo, S.B., Wheeler, T.D., McDermott, G.P., Zhu, J., et al. (2017). Massively parallel digital transcriptional profiling of single cells. Nat Commun 8, 14049.

Zhou, N., Pan, T., Zhang, J., Li, Q., Zhang, X., Bai, C., Huang, F., Peng, T., Liu, C., Tao, L., et al. (2016). Glycopeptide Antibiotics Potently Inhibit Cathepsin L in the Late Endosome/Lysosome and Block the Entry of Ebola Virus, Middle East Respiratory Syndrome Coronavirus (MERS-CoV), and Severe Acute Respiratory Syndrome Coronavirus (SARS-CoV). J Biol Chem 291, 9218-9232.

Zhou, X., Zhu, L., Lizarraga, R., and Chen, Y. (2017). Human Airway Epithelial Cells Direct Significant Rhinovirus Replication in Monocytic Cells by Enhancing ICAM1 Expression. Am J Respir Cell Mol Biol 57, 216-225.

Ziegler, C.a.A., Samuel J. and Nyquist, Sarah K. and Mbano, lan and Miao, Vincent N. and Cao, Yuming and Yousif, Ashraf S. and Bals, Julia and Hauser, Blake M. and Feldman, Jared and Muus, Christoph and Wadsworth II, Marc H. and Kazer, Samuel and Hughes, Travis K. and Doran, Benjamin and Gatter, G. James and Vukovic, Marko and Tzouanas, Constantine N. and Taliaferro, Faith and Guo, Zhiru and Wang, Jennifer P. and Dwyer, Daniel F. and Buchheit, Kathleen M. and Boyce, Joshua and Barrett, Nora A. and Laidlaw, Tanya M. and Carroll, Shaina L. and Colonna, Lucrezia and Tkachev, Victor and Yu, Alison and Zheng, Henqi Betty and Gideon, Hannah P. and Winchell, Caylin G. and Lin, Philana L. and Berger, Bonnie and Leslie, Alasdair and Flynn, JoAnne L. and Fortune, Sarah M. and Finberg, Robert W. and Kean, Leslie and Garber, Manuel and Schmidt, Aaron and Lingwood, Daniel and Shalek, Alex K. and Ordovas-Montanes, Jose and Lung Biological Network, HCA (2020). SARS-CoV2 Receptor ACE2 is an Interferon-Stimulated Gene in Human Airway Epithelial Cells and Is Enriched in Specific Cell Subsets Across Tissues. Cell.

Zou, X., Chen, K., Zou, J., Han, P., Hao, J., and Han, Z. (2020). Single-cell RNA-seq data analysis on the receptor ACE2 expression reveals the potential risk of different human organs vulnerable to 2019-nCoV infection. Front Med. 
Figure 1

A Human Lung snRNA-seq (46,500 nuclei)

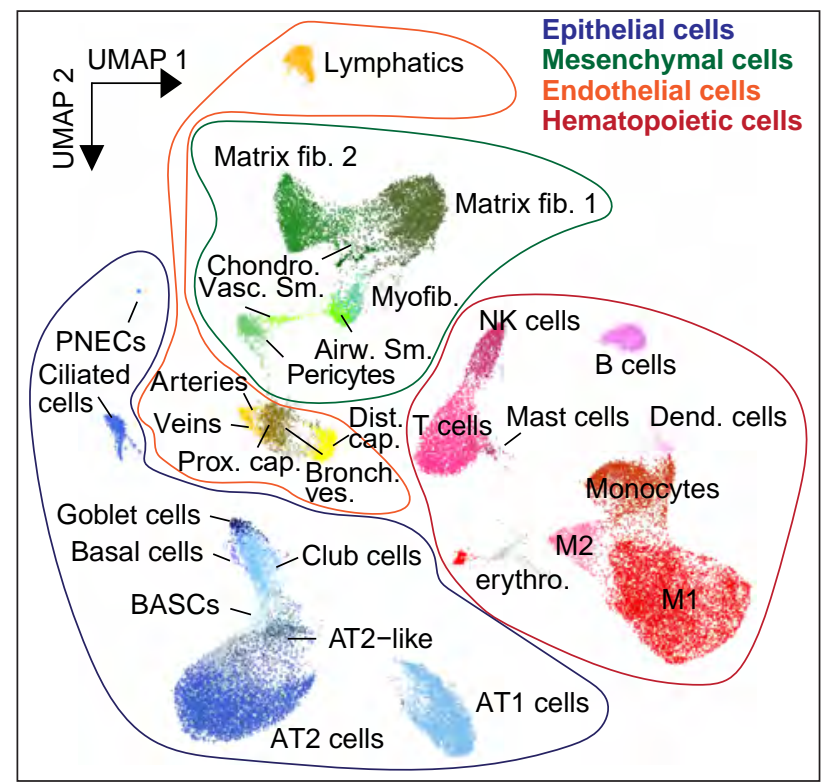

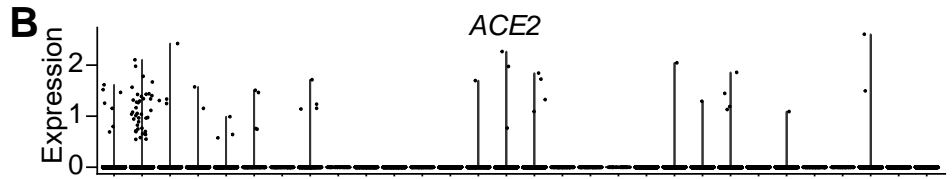

$\mathbf{C}_{\mathrm{C}_{4}}$
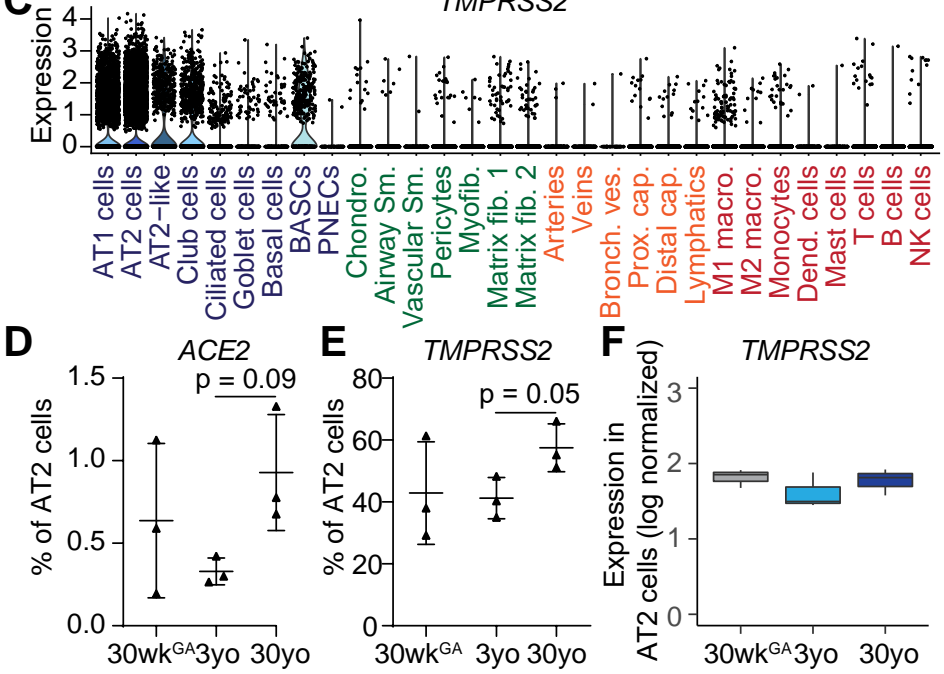
Figure 3

A
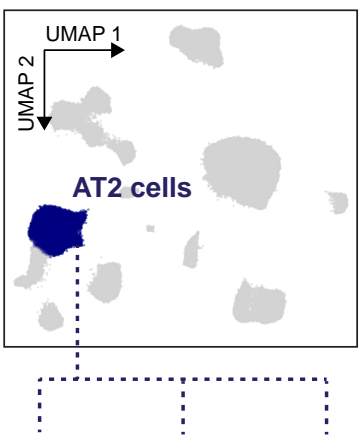

$30 \mathrm{wk}^{\mathrm{GA}} \quad 3 \mathrm{yo}$

30yo

5,633 nucl. 4,930 nucl. 3,562 nucl.

$n=3 \quad n=3 \quad n=3$

B
22,845 dynamic peaks in AT2 cells

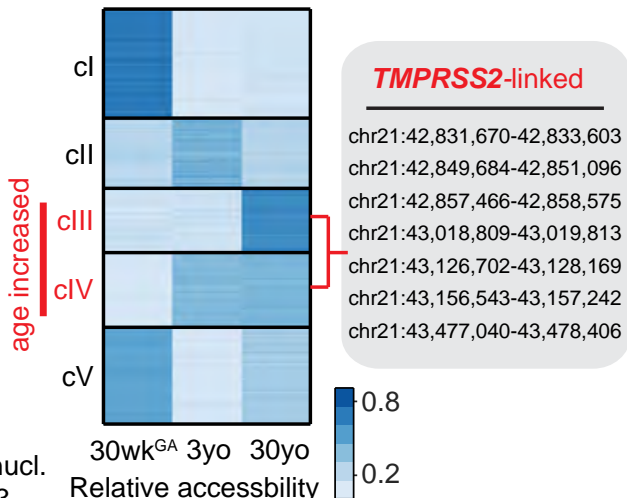

C

clll - 30yo-enriched

ֻٕ屯 positive regulation of ERBB signaling pathway

morph. of an epithelial fold

mast cell activation

activin receptor sign. path.

O regul. of prot. kin. A sign.

regul. of viral rel. from host cell

pos. regul. of EGF-activated receptor activity

abnormal mast cell number

decr. suscept. to bacterial inf. induced morbidity/mortality

pulmonary epithelial necrosis

$\begin{array}{lllllll}0 & 1 & 2 & 3 & 4 & 5 & 6\end{array}$ - $\log 10$ (Binomial P-value)

D

clll - 30yo-enriched

\begin{tabular}{|c|c|c|}
\hline \multicolumn{3}{|c|}{ TF MOTIF } \\
\hline JUN/FOS & 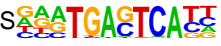 & \\
\hline CEBP & 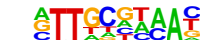 & 1 \\
\hline STAT & 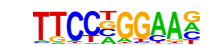 & $1 e-1$ \\
\hline NKX & 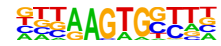 & $1 e-11$ \\
\hline FOXA & AGTAAACA & $1 e-9$ \\
\hline ETS & AПTTCCTC & $1 e-8$ \\
\hline RUNX & TGTGGCTTGCCA & $1 e-$ \\
\hline CTCF & CCCTCTAGAG & $1 e-1$ \\
\hline IRF & АCTTTC: & $1 \mathrm{e}-1$ \\
\hline
\end{tabular}

cIV - 30yo + 30yo

\begin{tabular}{|c|c|c|}
\hline TF & MOTIF & $p$-valu \\
\hline CEBP & A & $1 \mathrm{e}-286$ \\
\hline NKX & T⿱乛龰AAGTGC & $1 \mathrm{e}-134$ \\
\hline FOXA & 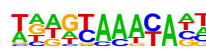 & $1 \mathrm{e}-105$ \\
\hline ETS & 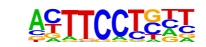 & $1 e-79$ \\
\hline UN/FOS & S TGAGTCAT & 1e-45 \\
\hline PROX1 & 1 A A & $1 \mathrm{e}-40$ \\
\hline RUNX & TETGGTIA & $1 \mathrm{e}-30$ \\
\hline CTCF & ACCAGGGGCCEC & $1 \mathrm{e}-18$ \\
\hline & GTAAATACTT & 1e-12 \\
\hline
\end{tabular}

\section{E $\quad \mathbf{F}$ \\ F}

TMPRSS2-linked peaks

(Access. in AT2 cells)
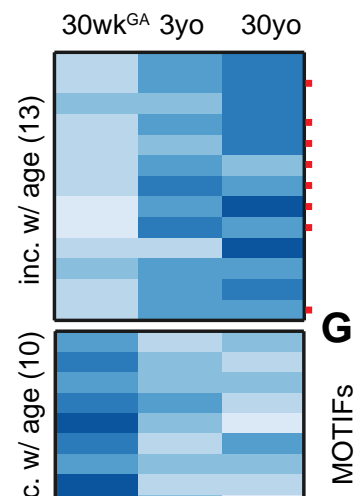

G 
Figure 4

A

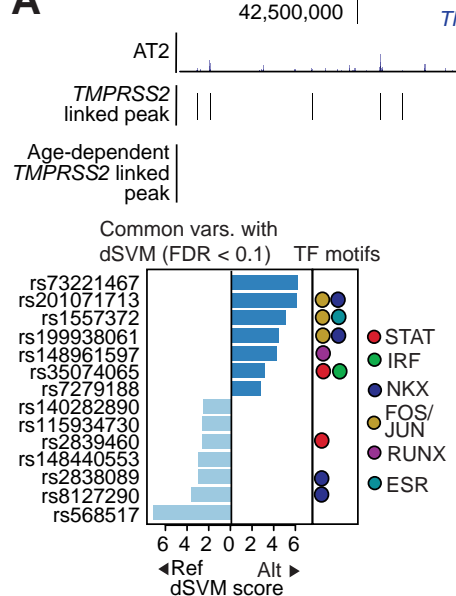

B

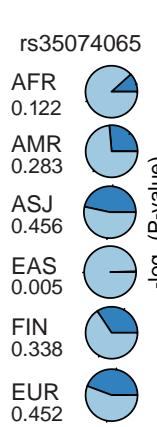

TMPRSS2 \#|W|
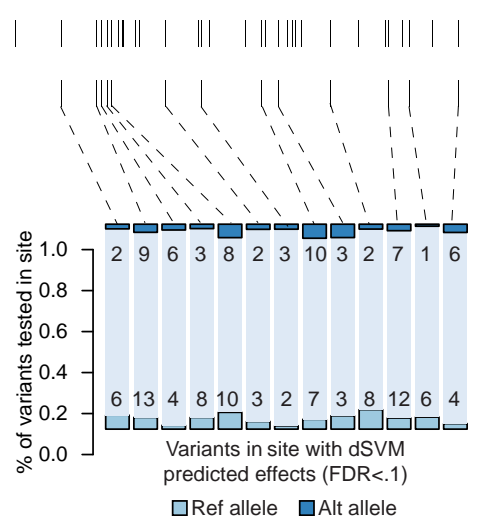

$\square$ Ref allele $\square$ Alt allele
$43,500,000$

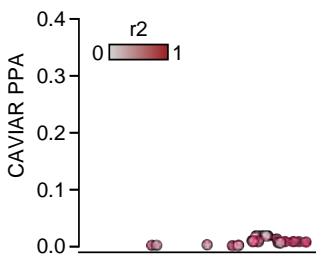

TMPRSS2 eQTL (GTEx v8 Lung)

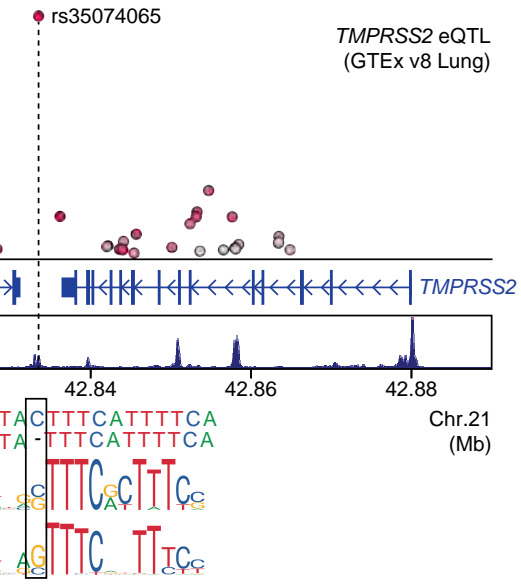

$E$

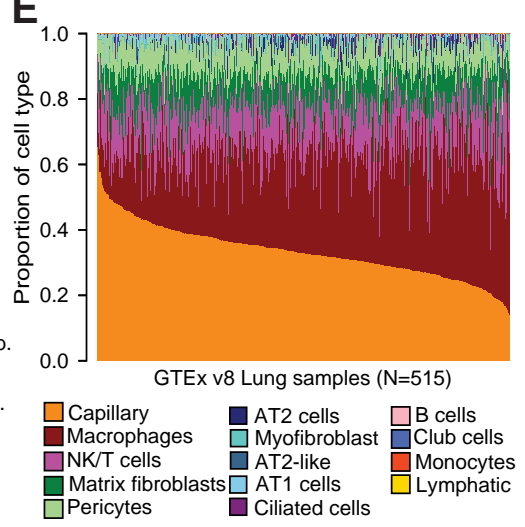

$\mathbf{F}$

TMPRSS2 expression (rs35074065)

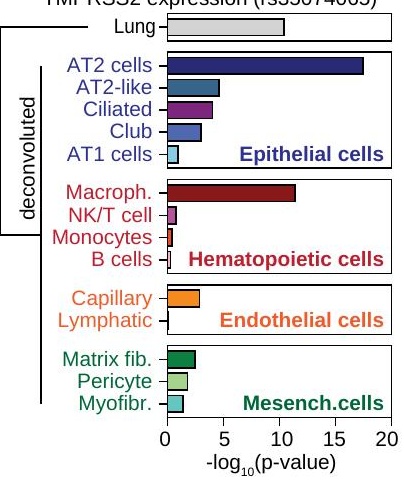

